\title{
An Adaptive Nonconforming Finite Element Algorithm for Laplace Eigenvalue Problem
}

\author{
Yuanyuan Yu, Yidu Yang, and Jiayu Han \\ School of Mathematics and Computer Science, Guizhou Normal University, Guiyang 550001, China \\ Correspondence should be addressed to Yidu Yang; ydyang@gznu.edu.cn
}

Received 11 January 2014; Revised 2 April 2014; Accepted 29 April 2014; Published 29 May 2014

Academic Editor: Agacik Zafer

Copyright (C) 2014 Yuanyuan Yu et al. This is an open access article distributed under the Creative Commons Attribution License, which permits unrestricted use, distribution, and reproduction in any medium, provided the original work is properly cited.

We establish Crouzeix-Raviart element adaptive algorithm based on Rayleigh quotient iteration and give its a priori/a posteriori error estimates. Our algorithm is performed under the package of Chen, and satisfactory numerical results are obtained.

\section{Introduction}

A posteriori error estimates and adaptive methods of finite element approximation for eigenvalue problems are topics attracting more attention from mathematical and physical fields; see, for example, [1-8]. Basically, there are the following three ways of combining adaptivity and eigenvalue problems in which the a posteriori error estimators are more or less the same but different in the problem solved in each iteration: (1) solving the original eigenvalue problem $a(u, v)=\lambda b(u, v)$ (see Algorithm 10). The convergence and optimality of this adaptive procedure were proved in [2]; (2) inverse iteration type (with or without correction). The convergence has been studied in $[1,6,7]$; (3) Shifted-inverse iteration type (see [811]).

The triangular Crouzeix-Raviart element (C-R element) was first introduced by Crouzeix and Raviart [12] in 1973 to solve the stationary Stokes equation. After that, many scholars developed and applied it to eigenvalue problems, for instance, [13-16] discussed a posteriori error estimates and the adaptive methods of the C-R element. C-R element has important properties; for example, Armentano and Durán [17] discovered and proved that the C-R element eigenvalues approximate the exact ones of the Laplace operator from below, which is a very important property in engineering and mechanics computing.

Based on the above work, this paper further discusses the third kind of adaptive methods of the C-R finite element method for eigenvalue problems and obtains the following new results:

(1) we establish a multiscale discretization scheme of the $\mathrm{C}-\mathrm{R}$ element based on Rayleigh quotient iteration and prove its convergence and a priori error estimates;

(2) we give residual type a posteriori error estimator for our adaptive algorithm, as well as its reliability and efficiency;

(3) we establish an adaptive algorithm (Algorithm 11), which is performed under the package of Chen (see [18]), and satisfactory numerical results are obtained.

As for the fundamental theory of finite elements and spectral approximation, we refer to [19-22].

Throughout this paper, $C$ denotes a positive constant independent of mesh parameter, which may not be the same constant in different places. For simplicity, we use the notation $a \leq b$ to mean that $a \leq C b, a=O(b)$ and to mean that $a \lesssim b$ and $b \lesssim a$.

\section{Preliminaries}

Consider Laplace eigenvalue problem

$$
-\Delta u=\lambda u \quad \text { in } \Omega, \quad u=0 \quad \text { on } \partial \Omega,
$$

where $\Omega \subset R^{2}$ is a polygonal domain with the maximum interior angle $\omega$. 
We denote the real order Sobolev spaces with norm $\|\cdot\|_{t}$ by $H^{t}(\Omega), H_{0}^{1}(\Omega)=\left\{v \in H^{1}(\Omega),\left.v\right|_{\partial \Omega}=0\right\}$. Let $b(\cdot, \cdot)$ and $\|\cdot\|_{0, \Omega}$ be the inner product and the norm in the space $L^{2}(\Omega)$, respectively.

The weak form of (1) is as follows: find $\lambda \in R, u \in H_{0}^{1}(\Omega)$, $u \neq 0$ such that

$$
a(u, v)=\lambda b(u, v), \quad \forall v \in H_{0}^{1}(\Omega),
$$

where

$$
a(u, v)=\int_{\Omega} \nabla u \cdot \nabla v, \quad b(u, v)=\int_{\Omega} u v .
$$

As we know, $a(\cdot, \cdot)$ is a symmetric, continuous, and $H_{0}^{1}(\Omega)$-elliptic bilinear form on $H_{0}^{1}(\Omega) \times H_{0}^{1}(\Omega)$, and $b(\cdot, \cdot)$ is a symmetric, continuous, and positive definite bilinear form on $L^{2}(\Omega) \times L^{2}(\Omega)$.

Define the operator $T: L^{2}(\Omega) \rightarrow H_{0}^{1}(\Omega)$, satisfying

$$
a(T g, v)=b(g, v), \quad \forall v \in H_{0}^{1}(\Omega) .
$$

Then, (2) has the equivalent operator form $T u=\lambda^{-1} u$, where the operators $T: H_{0}^{1}(\Omega) \rightarrow H_{0}^{1}(\Omega)$ and $T: L^{2}(\Omega) \rightarrow$ $L^{2}(\Omega)$ are self-adjoint and completely continuous.

Let $\pi_{h}=\{\kappa\}$ be a regular triangulation of the domain $\Omega, \varepsilon$ denote the set of all element edges in $\pi_{h}, \varepsilon(\Omega)$ denote the set of interior edges, $\varepsilon(\partial \Omega)$ denote the set of all boundary edges, and $N_{m}$ denote the set of the midside nodes $m_{e}$ of the edges $e \in \varepsilon$. For the set of midpoints of the edges $e \in \varepsilon(\partial \Omega)$, we use the notation $N_{m}(\partial \Omega)$; for any element $\kappa$, we let $\partial \kappa$ be the union set of edges of $\kappa$, and let $h_{\kappa}$ be the diameter of $\kappa$. The domain $\omega_{\kappa}$ consists of all elements sharing at least a side with $\kappa$. For any edge $e \in \varepsilon, h_{e}=|e|$ is the length of $e$, and $\vartheta_{e}=$ $\left(\vartheta_{1}, \vartheta_{2}\right)$ and $\tau_{e}=\left(-\vartheta_{2}, \vartheta_{1}\right)$ are unit outward normal vector and unit tangential vector, respectively.

Given a nonnegative integer $k$, the space $P_{k}(\kappa)$ consists of polynomials of total degree at most $k$ defined over $\kappa$. The C-R element space is given by $V_{h}=\left\{v \in L^{2}(\Omega),\left.v\right|_{\kappa} \in P_{1}(\kappa), \kappa \in\right.$ $\pi_{h}, v$ is continuous at each $m_{e} \in N_{m} \backslash N_{m}(\partial \Omega)$, and $v\left(m_{e}\right)=0$ for $\left.m_{e} \in N_{m}(\partial \Omega)\right\}$.

The C-R element approximation of (2) is given as follows: find $\lambda_{h} \in R, u_{h} \in V_{h}, u_{h} \neq 0$ such that

$$
a_{h}\left(u_{h}, v\right)=\lambda_{h} b\left(u_{h}, v\right), \quad \forall v \in V_{h},
$$

where

$$
a_{h}\left(u_{h}, v\right)=\sum_{\kappa \in \pi_{h}} \int_{\kappa} \nabla u_{h} \cdot \nabla v
$$

$a_{h}(\cdot, \cdot)$ is a symmetric, continuous, and $V_{h}$-elliptic bilinear form on $V_{h} \times V_{h} \cdot\|v\|_{h}=\sqrt{a_{h}(v, v)}$ is well known as the norm of the space $V_{h}$; let $D \subset \Omega, \bar{D}$ which consists of $\kappa \in \pi_{h}$,

$$
\begin{gathered}
a_{h, \kappa}(u, v)=\int_{\mathcal{\kappa}} u v, \quad a_{h, D}(u, v)=\sum_{\kappa \in \bar{D}} a_{h, \kappa}(u, v), \\
\|v\|_{h, D}=\sqrt{a_{h, D}(v, v) .}
\end{gathered}
$$

Define the operator $T_{h}: L^{2}(\Omega) \rightarrow V_{h}$, satisfying

$$
a_{h}\left(T_{h} g, v\right)=b(g, v), \quad \forall v \in V_{h} .
$$

Then, (5) has the equivalent operator form $T_{h} u_{h}=\lambda_{h}^{-1} u_{h}$, where the operators $T_{h}: V_{h} \rightarrow V_{h}$ and $T_{h}: L^{2}(\Omega) \rightarrow L^{2}(\Omega)$ are self-adjoint and completely continuous.

Suppose that $\lambda$ and $\lambda_{h}$ are the $k$ th eigenvalue of (2) and (5), respectively, and the algebraic multiplicity of $\lambda$ is equal to $q, \lambda=\lambda_{k}=\lambda_{k+1}=\cdots=\lambda_{k+q-1}$. Let $M\left(\lambda_{k}\right)$ be the space spanned by all eigenfunctions corresponding to $\lambda_{k}$, and let $M_{h}\left(\lambda_{k}\right)$ be the direct sum of eigenspaces corresponding to all eigenvalues of (5) that converge to $\lambda_{k}$. Let $\widehat{M}\left(\lambda_{k}\right)=\{v: v \in$ $\left.M\left(\lambda_{k}\right),\|v\|_{h}=1\right\}, \widehat{M}_{h}\left(\lambda_{k}\right)=\left\{v: v \in M_{h}\left(\lambda_{k}\right),\|v\|_{h}=1\right\}$.

Define

$$
\delta_{h}^{2}\left(\lambda_{k}\right)=\left\|\left.\left(T-T_{h}\right)\right|_{M\left(\lambda_{k}\right)}\right\|_{h}^{2}+\left\|\left.\left(T-T_{h}\right)\right|_{M\left(\lambda_{k}\right)}\right\|_{0} .
$$

Define $E_{h}(T f, v)=a_{h}(T f, v)-b(f, v)$, which is the consistent item of nonconforming finite element. Let $(\lambda, u)$ be an eigenpair of (2), and then

$$
E_{h}(u, v)=a_{h}(u, v)-b(\lambda u, v) .
$$

We need the lemmas as follows (see $[11,23])$.

Lemma 1 (see [11, Lemma 2.5]). Let $(\lambda, u)$ be an eigenpair of (2) and then, for any $w \in V_{h}$ with $\|w\|_{0} \neq 0$, the Rayleigh quotient $a_{h}(w, w) /\|w\|_{0}^{2}$ satisfies

$$
\frac{a_{h}(w, w)}{\|w\|_{0}^{2}}-\lambda=\frac{\|w-u\|_{h}^{2}}{\|w\|_{0}^{2}}-\lambda \frac{\|w-u\|_{0}^{2}}{\|w\|_{0}^{2}}+2 \frac{E_{h}(u, w)}{b(w, w)} .
$$

Lemma 2 (see [11, Lemma 2.4] and [23, Lemma 2.3]). Let $\lambda_{k, h}$ and $\lambda_{k}$ be the $k$ th eigenvalue of (5) and (2), respectively. Then,

$$
\left|\lambda_{k, h}-\lambda_{k}\right| \lesssim\left\|\left.\left(T-T_{h}\right)\right|_{M\left(\lambda_{k}\right)}\right\|_{0} .
$$

For any eigenfunction $u_{k, h}$ corresponding to $\lambda_{k, h}$, satisfying $\left\|u_{k, h}\right\|_{h}=1$, there exists $u_{k} \in M\left(\lambda_{k}\right)$ such that

$$
\begin{aligned}
& \left\|u_{k, h}-u_{k}\right\|_{0} \lesssim\left\|\left.\left(T-T_{h}\right)\right|_{M\left(\lambda_{k}\right)}\right\|_{0}, \\
& \left\|u_{k, h}-u_{k}\right\|_{h} \leq \lambda_{k}\left\|T u_{k}-T_{h} u_{k}\right\|_{h}+C\left\|\left.\left(T-T_{h}\right)\right|_{M\left(\lambda_{k}\right)}\right\|_{0}
\end{aligned}
$$

For any $u_{k} \in \widehat{M}\left(\lambda_{k}\right)$, there exists $u_{h} \in M_{h}\left(\lambda_{k}\right)$ such that

$$
\left\|u_{h}-u_{k}\right\|_{h} \lesssim\left\|\left.\left(T-T_{h}\right)\right|_{M\left(\lambda_{k}\right)}\right\|_{h}+\left\|\left.\left(T-T_{h}\right)\right|_{M\left(\lambda_{k}\right)}\right\|_{0} .
$$

\section{A Priori Error Estimates for Multiscale Discretization Scheme}

In this section, we will discuss a priori error estimates of the C-R finite element multiscale discretization scheme based on the shift-inverse power method. Let $\left\{\pi_{h_{i}}\right\}_{0}^{l}$ be a family of shape-regular meshes and let $\left\{V_{h_{i}}\right\}_{0}^{l}$ be the C-R finite element spaces defined on $\left\{\pi_{h_{i}}\right\}_{0}^{l}$. Besides, let $\pi_{H}=\pi_{h_{0}}, V_{H}=V_{h_{0}}$.

The following condition results from $[10,24]$. 
Condition 1. There exists a properly small positive number $\epsilon$, $t_{i} \in(1,3-\epsilon], i=1,2, \ldots$, such that $\delta_{h_{i}}(\lambda)=O\left(\delta_{h_{i-1}}(\lambda)^{t_{i}}\right)$, $\delta_{h_{i}}(\lambda) \rightarrow 0(i \rightarrow \infty)$. [11]).

The following scheme is proposed by Yang and Bi (see

Scheme 3 (multiscale discretization scheme). Consider the following steps.

Step 1. Solve (5) on $V_{H}$ : find $\left(\lambda_{H}, u_{H}\right) \in R \times V_{H}$ such that $\left\|u_{H}\right\|_{H}=1$ and

$$
a_{H}\left(u_{H}, \psi\right)=\lambda_{H} b\left(u_{H}, \psi\right), \quad \forall \psi \in V_{H} .
$$

Step 2. Execute $u^{h_{0}} \Leftarrow u_{H}, \lambda^{h_{0}} \Leftarrow \lambda_{H}, i \Leftarrow 1$.

Step 3. Solve a linear system on $V_{h_{i}}$ : find $u^{\prime} \in V_{h_{i}}$ such that

$$
a_{h_{i}}\left(u^{\prime}, \psi\right)-\lambda^{h_{i-1}} b\left(u^{\prime}, \psi\right)=b\left(u^{h_{i-1}}, \psi\right), \quad \forall \psi \in V_{h_{i}} .
$$

Set $u^{h_{i}}=u^{\prime} /\left\|u^{\prime}\right\|_{h_{i}}$.

Step 4. Compute the Rayleigh quotient

$$
\lambda^{h_{i}}=\frac{a_{h_{i}}\left(u^{h_{i}}, u^{h_{i}}\right)}{b\left(u^{h_{i}}, u^{h_{i}}\right)} .
$$

Step 5. If $i=l$, then output $\left(\lambda^{h_{l}}, u^{h_{l}}\right)$, stop. Else, $i \Leftarrow i+1$, and return to Step 3.

Let $\left(\lambda_{H}, u_{H}\right)$ be the $k$ th eigenpair of (16), and then $\left(\lambda^{h_{l}}, u^{h_{l}}\right)$ derived from Scheme 3 is the $k$ th eigenpair approximation of (5).

In the sequel, we also denote $\left(\lambda_{H}, u_{H}\right)=\left(\lambda_{k, H}, u_{k, H}\right)$, $\left(\lambda^{h_{l}}, u^{h_{l}}\right)=\left(\lambda_{k}^{h_{l}}, u_{k}^{h_{l}}\right)$.

Lemma 4 (see [11, Lemma 3.1]). For any nonzero $u, v \in V_{h}+$ $H_{0}^{1}(\Omega)$,

$$
\begin{aligned}
& \left\|\frac{u}{\|u\|_{h}}-\frac{v}{\|v\|_{h}}\right\|_{h} \leq 2 \frac{\|u-v\|_{h}}{\|u\|_{h}}, \\
& \left\|\frac{u}{\|u\|_{h}}-\frac{v}{\|v\|_{h}}\right\|_{h} \leq 2 \frac{\|u-v\|_{h}}{\|v\|_{h}} .
\end{aligned}
$$

Denote $\operatorname{dist}(u, S)=\inf _{v \in S}\|u-v\|_{h}$.

Our analysis is based on the following crucial property of the shifted-inverse iteration in finite element method (see Lemma 4.2 of [24]), which is a development of Theorem 3.2 in [11]. Let $M=\left(1 / \lambda_{k}\right)=M\left(\lambda_{k}\right), M_{h}=\left(1 / \lambda_{k}\right)=M_{h}\left(\lambda_{k}\right)$.

Lemma 5 (see [24, Lemma 4.2]). Let $\mu_{k}=1 / \lambda_{k}$ and $\mu_{k, h}=$ $1 / \lambda_{k, h}$ be the kth eigenvalue of $T$ and $T_{h}$, respectively, $\left(\mu_{0}, u_{0}\right)$ be an approximation for the eigenpair $\left(\mu_{k}, u_{k}\right)$, where $\mu_{0}$ is not an eigenvalue of $T_{h}$, and $u_{0} \in V_{h}$ with $\left\|u_{0}\right\|_{h}=1$. Suppose that

(C1) $\operatorname{dist}\left(u_{0}, M_{h}\left(\mu_{k}\right)\right) \leq 1 / 2$;
(C2) $\left|\mu_{0}-\mu_{j, h}\right| \geq \rho / 2$ for $j \neq k, k+1, \ldots, k+q-1$, where $\rho=\min _{\mu_{j} \neq \mu_{k}}\left|\mu_{j}-\mu_{k}\right|$ is the separation constant of the eigenvalue $\mu_{k}$;

(C3) $u^{\prime} \in V_{h}, u_{k}^{h} \in V_{h}$ satisfy

$$
\left(\mu_{0}-T_{h}\right) u^{\prime}=u_{0}, \quad u_{k}^{h}=\frac{u^{\prime}}{\left\|u^{\prime}\right\|_{h}} .
$$

Then,

$$
\begin{aligned}
& \operatorname{dist}\left(u_{k}^{h}, M_{h}\left(\mu_{k}\right)\right) \\
& \quad \leq \frac{4}{\rho} \max _{k \leq j \leq k+q-1}\left|\mu_{0}-\mu_{j, h}\right| \operatorname{dist}\left(u_{0}, M_{h}\left(\mu_{k}\right)\right) .
\end{aligned}
$$

Let us construct the interpolation postprocessing operator $I_{h}^{c}: V_{h} \rightarrow V_{h} \cap H_{0}^{1}(\Omega)$ (see [25]): on the vertex $z$ of elements,

$$
\left(I_{h}^{c} u_{h}\right)(z)= \begin{cases}0, & z \in \partial \Omega, \\ \left.\frac{1}{J_{z}} \sum_{\kappa \in \omega_{z}} u_{h}\right|_{\mathcal{K}}(z), & z \notin \partial \Omega,\end{cases}
$$

where $J_{z}$ is the number of elements containing the vertex $z$ and $\omega_{z}$ is the union of elements containing the vertex $z$.

Lemma 6. Suppose that Condition 1 holds and $H$ is properly small. Let $\left(\lambda_{k}^{h_{1}}, u_{k}^{h_{1}}\right)$ be obtained by Scheme 3 for $l=1$, and then there exists $u_{k} \in M\left(\lambda_{k}\right)$ such that

$$
\begin{gathered}
\left\|u_{k}^{h_{1}}-u_{k}\right\|_{h_{1}} \leq C\left(\delta_{H}^{3}\left(\lambda_{k}\right)+\delta_{h_{1}}\left(\lambda_{k}\right)\right), \\
\left|\lambda_{k}^{h_{1}}-\lambda_{k}\right| \leq C\left(\delta_{H}^{6}\left(\lambda_{k}\right)+\delta_{h_{1}}^{2}\left(\lambda_{k}\right)\right) .
\end{gathered}
$$

Proof. Based on the proof of Theorem 5.1 in [11] and Lemma 5, we deduce that

$$
\begin{aligned}
& \left\|u_{k}^{h_{1}}-u_{k}\right\|_{h_{1}} \\
& \quad \leq C\left(\left\|\left.\left(T-T_{H}\right)\right|_{M\left(\lambda_{k}\right)}\right\|_{0}^{2}+\left\|\left.\left(T-T_{h_{1}}\right)\right|_{M\left(\lambda_{k}\right)}\right\|_{h_{1}}\right) \\
& \quad \leq \delta_{H}^{4}\left(\lambda_{k}\right)+\delta_{h_{1}}\left(\lambda_{k}\right),
\end{aligned}
$$

and thus (23) holds. Using Strang Lemma and Lemma 3.1 of [25], we deduce that

$$
\begin{aligned}
& E_{h_{1}}\left(u_{k}, u_{k}^{h_{1}}\right) \\
& \quad=E_{h_{1}}\left(u_{k}, u_{k}^{h_{1}}-I_{h_{1}}^{c} u_{k}^{h_{1}}\right) \\
& \quad \lesssim\left\|u_{k}-T_{h_{1}}\left(\lambda_{k} u_{k}\right)\right\|_{h_{1}}\left\|u_{k}^{h_{1}}-I_{h_{1}}^{c} u_{k}^{h_{1}}\right\|_{h_{1}} \\
& \quad \lesssim\left\|u_{k}-T_{h_{1}}\left(\lambda_{k} u_{k}\right)\right\|_{h_{1}}\left\|u_{k}^{h_{1}}-u_{k}\right\|_{h_{1}} \lesssim \delta_{h_{1}}^{2}\left(\lambda_{k}\right) .
\end{aligned}
$$


From the above formula and (5.2) in [11], we get

$$
\begin{aligned}
& \left|\lambda_{k}^{h_{1}}-\lambda_{k}\right| \\
& \leq C\left(\left\|\left.\left(T-T_{H}\right)\right|_{M\left(\lambda_{k}\right)}\right\|_{0}^{4}+\left\|\left.\left(T-T_{h_{1}}\right)\right|_{M\left(\lambda_{k}\right)}\right\|_{h_{1}}^{2}\right. \\
& \left.+2 \frac{E_{h_{1}}\left(u_{k}, u_{k}^{h_{1}}\right)}{b\left(u_{k}^{h_{1}}, u_{k}^{h_{1}}\right)}\right) \\
& \leq \delta_{H}^{6}\left(\lambda_{k}\right)+\delta_{h_{1}}^{2}\left(\lambda_{k}\right),
\end{aligned}
$$

and thus (24) holds.

Based on $[10,11,24]$, we will prove the following Theorems 7 and 8 for Scheme 3.

Theorem 7. Let $\left(\lambda^{h_{l}}, u^{h_{l}}\right)$ be an approximate eigenpair obtained by Scheme 3, and $u^{h_{l-1}}$ and $\lambda^{h_{l-1}}$ approximate $\bar{u} \epsilon$ $\widehat{M}(\lambda)$ and $\lambda$, respectively, and $\left\|u^{h_{l-1}}-\bar{u}\right\|_{h_{l-1}} \lesssim \delta_{h_{l-1}}(\lambda), \mid \lambda^{h_{l-1}}-$ $\lambda \mid \lesssim \delta_{h_{l-1}}^{2}(\lambda)$. Suppose that $H$ is properly small and Condition 1 holds. Then, there exists $u \in M(\lambda)$ such that

$$
\begin{gathered}
\left\|u-u^{h_{l}}\right\|_{h_{l}, D}=\left\|\left(T-T_{h_{l}}\right)\left(\lambda^{h_{l}} u^{h_{l}}\right)\right\|_{h_{l}, D}+\|R\|_{h_{l}, D}, \\
\left|\lambda^{h_{l}}-\lambda\right| \lesssim \delta_{h_{l}}^{2}(\lambda), \\
\left\|u^{h_{l}}-T_{h_{l}}\left(\lambda^{h_{l}} u^{h_{l}}\right)\right\|_{h_{l}, D} \leqslant \delta_{h_{l-1}}^{3}(\lambda)+\delta_{h_{l}}^{2}(\lambda),
\end{gathered}
$$

where $\|R\|_{h_{l}, D} \lesssim \delta_{h_{l-1}}^{3}(\lambda)+\delta_{h_{l}}^{2}(\lambda)$.

Proof. Let $\mu_{0}=1 / \lambda^{h_{l-1}}, u_{0}=\lambda^{h_{l-1}} T_{h_{l}} u^{h_{l-1}} /\left\|\lambda^{h_{l-1}} T_{h_{l}} u^{h_{l-1}}\right\|_{h_{l}}$. Since $\bar{u} \in \widehat{M}(\lambda)$, by calculation, we get

$$
\begin{aligned}
& \left\|\lambda^{h_{l-1}} T_{h_{l}} u^{h_{l-1}}-\bar{u}\right\|_{h_{l}} \\
& =\left\|\lambda^{h_{l-1}} T_{h_{l}} u^{h_{l-1}}-\lambda T \bar{u}\right\|_{h_{l}} \\
& \leq\left|\lambda^{h_{l-1}}-\lambda\right|\left\|T_{h_{l}} u^{h_{l-1}}\right\|_{h_{l}}+\lambda\left\|T_{h_{l}}\left(u^{h_{l-1}}-\bar{u}\right)\right\|_{h_{l}} \\
& \quad+\lambda\left\|\left(T_{h_{l}}-T\right) \bar{u}\right\|_{h_{l}} .
\end{aligned}
$$

From the definition of $T_{h_{l}}$, it is easy to know that

$$
\left\|T_{h_{l}} v\right\|_{h_{l}} \leq C\|v\|_{0}, \quad \forall v \in L^{2}(\Omega) .
$$

From $a_{h_{l}}\left(T_{h_{l}} v_{h_{l}}, v_{h_{l}}\right)=b\left(v_{h_{l}}, v_{h_{l}}\right)$, we get

$$
\left\|v_{h_{l}}\right\|_{0}^{2} \lesssim\left\|T_{h_{l}} v_{h_{l}}\right\|_{h_{l}}\left\|v_{h_{l}}\right\|_{h_{l}} \lesssim\left\|v_{h_{l}}\right\|_{0}\left\|v_{h_{l}}\right\|_{h_{l}},
$$

and thus

$$
\left\|v_{h_{l}}\right\|_{0} \leqslant\left\|v_{h_{l}}\right\|_{h_{l}}, \quad \forall v_{h_{l}} \in V_{h_{l}}
$$

By Lemma 3.1 in [25], we get that

$$
\begin{gathered}
\left\|u^{h_{l-1}}-I_{h_{l-1}}^{c} u^{h_{l-1}}\right\|_{0} \lesssim\left\|u^{h_{l-1}}-I_{h_{l-1}}^{c} u^{h_{l-1}}\right\|_{h_{l-1}}, \\
\left\|u^{h_{l-1}}-I_{h_{l-1}}^{c} u^{h_{l-1}}\right\|_{h_{l-1}} \lesssim\left\|u^{h_{l-1}}-\bar{u}\right\|_{h_{l-1}} .
\end{gathered}
$$

Thus,

$$
\begin{aligned}
& \left\|u^{h_{l-1}}-\bar{u}\right\|_{0} \\
& \quad \leq\left\|u^{h_{l-1}}-I_{h_{l-1}}^{c} u^{h_{l-1}}\right\|_{0}+\left\|I_{h_{l-1}}^{c} u^{h_{l-1}}-\bar{u}\right\|_{0} \\
& \quad \leq\left\|u^{h_{l-1}}-I_{h_{l-1}}^{c} u^{h_{l-1}}\right\|_{h_{l-1}}+\left\|I_{h_{l-1}}^{c} u^{h_{l-1}}-\bar{u}\right\|_{h_{l-1}} \\
& \quad \leq\left\|u^{h_{l-1}}-\bar{u}\right\|_{h_{l-1}} .
\end{aligned}
$$

Using the above formula and (31), we can deduce that

$$
\begin{aligned}
\left\|\lambda^{h_{l-1}} T_{h_{l}} u^{h_{l-1}}-\bar{u}\right\|_{h_{l}} \lesssim & \left|\lambda^{h_{l-1}}-\lambda\right|+\lambda\left\|u^{h_{l-1}}-\bar{u}\right\|_{0} \\
& +\lambda\left\|\left.\left(T_{h_{l}}-T\right)\right|_{M(\lambda)}\right\|_{h_{l}} \\
\lesssim & \delta_{h_{l-1}}(\lambda) .
\end{aligned}
$$

Using Lemma 4, we get

$$
\begin{aligned}
\operatorname{dist}\left(u_{0}, \widehat{M}(\lambda)\right) & \leq\left\|u_{0}-\bar{u}\right\|_{h_{l}} \leq 2\left\|\lambda^{h_{l-1}} T_{h_{l}} u^{h_{l-1}}-\bar{u}\right\|_{h_{l}} \\
& \leq \delta_{h_{l-1}}(\lambda) .
\end{aligned}
$$

Using triangle inequality and (15), we have

$$
\operatorname{dist}\left(u_{0}, M_{h_{l}}(\lambda)\right) \leq \operatorname{dist}\left(u_{0}, \widehat{M}(\lambda)\right)+\delta_{h_{l}}(\lambda) \text {. }
$$

From (12), for $j=k, k+1, \ldots, k+q-1$, we have

$$
\left|\mu_{0}-\mu_{j, h_{l}}\right|=\left|\frac{\lambda^{h_{l-1}}-\lambda+\lambda-\lambda_{j, h_{l}}}{\lambda_{j, h_{l}} \lambda^{h_{l-1}}}\right| \lesssim\left|\lambda^{h_{l-1}}-\lambda\right|+\delta_{h_{l}}^{2}(\lambda) .
$$

Noticing that $H$ is small enough and Condition 1 holds, then by (38) and (39), we can obtain

$$
\operatorname{dist}\left(u_{0}, M_{h_{l}}(\lambda)\right) \leq \frac{1}{2}
$$

Since $\rho$ is the separation constant, $H$ is small enough, and Condition 1 holds, we have

$$
\left|\mu_{0}-\mu_{j, h_{l}}\right| \geq \frac{\rho}{2}, \quad j \neq k, k+1, \ldots, k+q-1 .
$$

From the definition of $T_{h_{l}}$, we can see that Step 3 in Scheme $3(i=l)$ is equivalent to

$$
\begin{aligned}
& a_{h_{l}}\left(u^{\prime}, \psi\right)-\lambda^{h_{l-1}} a_{h_{l}}\left(T_{h_{l}} u^{\prime}, \psi\right) \\
& \quad=a_{h_{l}}\left(T_{h_{l}} u^{h_{l-1}}, \psi\right), \quad \forall \psi \in V_{h_{l}},
\end{aligned}
$$


where $u^{h_{l}}=u^{\prime} /\left\|u^{\prime}\right\|_{h_{l}}$; that is,

$$
\left(\frac{1}{\lambda^{h_{l-1}}}-T_{h_{l}}\right) u^{\prime}=\frac{1}{\lambda^{h_{l-1}}} T_{h_{l}} u^{h_{l-1}}, \quad u^{h_{l}}=\frac{u^{\prime}}{\left\|u^{\prime}\right\|_{h_{l}}} .
$$

Noticing that $\left(1 / \lambda^{h_{l-1}}\right) T_{h_{l}} u^{h_{l-1}}=\left\|\left(1 / \lambda^{h_{l-1}}\right) T_{h_{l}} u^{h_{l-1}}\right\|_{h_{l}} u_{0}$ differs from $u_{0}$ by only a constant, then Step 3 is equivalent to

$$
\left(\frac{1}{\lambda^{h_{l-1}}}-T_{h_{l}}\right) u=u_{0}, \quad u^{h_{l}}=\frac{u}{\|u\|_{h_{l}}} .
$$

From the above formulae, (41), (42), and (45), we can see that the conditions in Lemma 5 hold; therefore, substituting (39) and (40) into (21), we derive

$$
\begin{aligned}
\operatorname{dist} & \left(u^{h_{l}}, \widehat{M}_{h_{l}}(\lambda)\right) \\
\quad & \leqslant\left(\left|\lambda^{h_{l-1}}-\lambda\right|+\delta_{h_{l}}^{2}(\lambda)\right)\left(\operatorname{dist}\left(u_{0}, \widehat{M}(\lambda)\right)+\delta_{h_{l}}(\lambda)\right) \\
\quad & \leqslant \delta_{h_{l-1}}^{3}+\delta_{h_{l-1}}^{2} \delta_{h_{l}}(\lambda) \lesssim \delta_{h_{l-1}}^{3}(\lambda) .
\end{aligned}
$$

Let eigenfunctions $\left\{u_{j, h_{l}}\right\}_{k}^{k+q-1}$ be an orthonormal basis of $M_{h_{l}}(\lambda)$ in the sense of inner product $a_{h_{l}}(\cdot, \cdot)$ and then

$$
\operatorname{dist}\left(u^{h_{l}}, M_{h_{l}}(\lambda)\right)=\left\|u^{h_{l}}-\sum_{j=k}^{k+q-1} a_{h_{l}}\left(u^{h_{l}}, u_{j, h_{l}}\right) u_{j, h_{l}}\right\|_{h_{l}} .
$$

Let

$$
u^{*}=\sum_{j=k}^{k+q-1} a_{h_{l}}\left(u^{h_{l}}, u_{j, h_{l}}\right) u_{j, h_{l}}
$$

and then it follows directly from (46) that

$$
\left\|u^{h_{l}}-u^{*}\right\|_{h_{l}} \leq \operatorname{dist}\left(u^{h_{l}}, \widehat{M}_{h_{l}}(\lambda)\right) \lesssim \delta_{h_{l-1}}^{3}(\lambda) .
$$

By Lemma 2, there exists $\left\{u_{j}^{0}\right\}_{k}^{k+q-1} \subset \widehat{M}(\lambda)$ so that $u_{j, h_{l}}-u_{j}^{0}$ satisfies (14).

Let

$$
u=\sum_{j=k}^{k+q-1} a_{h_{l}}\left(u^{h_{l}}, u_{j, h_{l}}\right) u_{j}^{0}
$$

Then, $u \in M(\lambda)$, and

$$
u-u^{*}=\sum_{j=k}^{k+q-1} a_{h_{l}}\left(u^{h_{l}}, u_{j, h_{l}}\right)\left(u_{j}^{0}-u_{j, h_{l}}\right) .
$$

By calculation,

$$
\begin{aligned}
u_{j}^{0}-u_{j, h_{l}}= & \lambda_{j} T u_{j}^{0}-\lambda_{j, h_{l}} T_{h_{l}} u_{j, h_{l}} \\
= & \lambda_{j} T u_{j}^{0}-\lambda_{j, h_{l}} T u_{j}^{0}+\lambda_{j, h_{l}} T u_{j}^{0}-\lambda_{j, h_{l}} T u_{j, h_{l}} \\
& +\lambda_{j, h_{l}} T u_{j, h_{l}}-\lambda_{j, h_{l}} T_{h_{l}} u_{j, h_{l}} \\
= & \left(\lambda_{j}-\lambda_{j, h_{l}}\right) T u_{j}^{0}+\lambda_{j, h_{l}} T\left(u_{j}^{0}-u_{j, h_{l}}\right) \\
& +\lambda_{j, h_{l}}\left(T-T_{h_{l}}\right) u_{j, h_{l}} \\
= & \lambda_{j, h_{l}}\left(T-T_{h_{l}}\right) u_{j, h_{l}}+R_{j}^{\prime},
\end{aligned}
$$

where $R_{j}^{\prime}=\left(\lambda_{j}-\lambda_{j, h_{l}}\right) T u_{j}^{0}+\lambda_{j, h_{l}} T\left(u_{j}^{0}-u_{j, h_{l}}\right)$.
From (12) and (13), we deduce that

$$
\left\|R_{j}^{\prime}\right\|_{h_{l}}=\left\|\left(\lambda_{j}-\lambda_{j, h_{l}}\right) T u_{j}^{0}+\lambda_{j, h_{l}} T\left(u_{j}^{0}-u_{j, h_{l}}\right)\right\|_{h_{l}} \leqslant \delta_{h_{l}}^{2}(\lambda) .
$$

Substituting (52) into (51), we have

$$
\begin{aligned}
u-u^{*}= & \sum_{j=k}^{k+q-1} a_{h_{l}}\left(u^{h_{l}}, u_{j, h_{l}}\right)\left(\lambda_{j, h_{l}}\left(T-T_{h_{l}}\right) u_{j, h_{l}}+R_{j}^{\prime}\right) \\
= & \lambda_{j, h_{l}}\left(T-T_{h_{l}}\right) \sum_{j=k}^{k+q^{-1}} a_{h_{l}}\left(u^{h_{l}}, u_{j, h_{l}}\right) u_{j, h_{l}} \\
& +\sum_{j=k}^{k+q-1} a_{h_{l}}\left(u^{h_{l}}, u_{j, h_{l}}\right) R_{j}^{\prime} \\
= & \left(T-T_{h_{l}}\right)\left(\lambda_{j, h_{l}} u^{*}\right)+\sum_{j=k}^{k+q-1} a_{h_{l}}\left(u^{h_{l}}, u_{j, h_{l}}\right) R_{j}^{\prime} \\
= & \left(T-T_{h_{l}}\right)\left(\lambda_{j, h_{l}} u^{h_{l}}\right)+\lambda_{j, h_{l}}\left(T-T_{h_{l}}\right)\left(u^{*}-u^{h_{l}}\right) \\
& +\sum_{j=k}^{k+q-1} a_{h_{l}}\left(u^{h_{l}}, u_{j, h_{l}}\right) R_{j}^{\prime} .
\end{aligned}
$$

Let

$$
\begin{aligned}
R^{\prime}= & \lambda_{j, h_{l}}\left(T-T_{h_{l}}\right)\left(u^{*}-u^{h_{l}}\right)+\sum_{j=k}^{k+q-1} a_{h_{l}}\left(u^{h_{l}}, u_{j, h_{l}}\right) R_{j}^{\prime} \\
& +u^{*}-u^{h_{l}} .
\end{aligned}
$$

By the above two equalities, we obtain

$$
u-u^{h_{l}}=u-u^{*}+u^{*}-u^{h_{l}}=\left(T-T_{h_{l}}\right)\left(\lambda_{j, h_{l}} u^{h_{l}}\right)+R^{\prime} .
$$

From (49) and (53), we have

$$
\begin{aligned}
\left\|R^{\prime}\right\|_{h_{l}, D}= & \| \lambda_{j, h_{l}}\left(T-T_{h_{l}}\right)\left(u^{*}-u^{h_{l}}\right) \\
& \quad+\sum_{j=k}^{k+q-1} a_{h_{l}}\left(u^{h_{l}}, u_{j, h_{l}}\right) R_{j}^{\prime}+u^{*}-u^{h_{l}} \|_{h_{l}, D} \\
\leq & \mid \lambda_{j, h_{l}}\left\|T-T_{h_{l}}\right\|_{h_{l}, D}\left\|u^{*}-u^{h_{l}}\right\|_{h_{l}, D} \\
& +\left\|\sum_{j=k}^{k+q-1} a_{h_{l}}\left(u^{h_{l}}, u_{j, h_{l}}\right) R_{j}^{\prime}\right\|_{h_{l}, D}+\left\|u^{*}-u^{h_{l}}\right\|_{h_{l}, D} \\
\leq & \left\|u^{*}-u^{h_{l}}\right\|_{h_{l}, D}+\left\|\sum_{j=k}^{k+q^{\prime}-1} a_{h_{l}}\left(u^{h_{l}}, u_{j, h_{l}}\right) R_{j}^{\prime}\right\|_{h_{l}, D} \\
\leq & \delta_{h_{l-1}}^{3}(\lambda)+\delta_{h_{l}}^{2}(\lambda) .
\end{aligned}
$$


Therefore,

$$
\begin{aligned}
\left\|u-u^{h_{l}}\right\|_{h_{l}, D} & =\left\|\left(T-T_{h_{l}}\right)\left(\lambda_{j, h_{l}} u^{h_{l}}\right)\right\|_{h_{l}, D}+\left\|R^{\prime}\right\|_{h_{l}, D} \\
& \lesssim \delta_{h_{l}}(\lambda) .
\end{aligned}
$$

By Lemma 1, we have

$$
\begin{aligned}
\frac{a_{h}\left(u^{h_{l}}, u^{h_{l}}\right)}{\left\|u^{h_{l}}\right\|_{0}^{2}}-\lambda= & \frac{\left\|u^{h_{l}}-u\right\|_{h_{l}}^{2}}{\left\|u^{h_{l}}\right\|_{0}^{2}}-\lambda \frac{\left\|u^{h_{l}}-u\right\|_{0}^{2}}{\left\|u^{h_{l}}\right\|_{0}^{2}} \\
& +2 \frac{E_{h}\left(u, u^{h_{l}}\right)}{b\left(u^{h_{l}}, u^{h_{l}}\right)} .
\end{aligned}
$$

Since $I_{h_{l}}^{c} u^{h_{l}} \in C^{0}(\bar{\Omega})$, using Strang Lemma and Lemma 3.1 of [25], we deduce that

$$
\begin{aligned}
E_{h_{l}}\left(u, u^{h_{l}}\right) & =E_{h_{l}}\left(u, u^{h_{l}}-I_{h_{l}}^{c} u^{h_{l}}\right) \\
& \lesssim\left\|u-T_{h_{l}}(\lambda u)\right\|_{h_{l}}\left\|u^{h_{l}}-I_{h_{l}}^{c} u^{h_{l}}\right\|_{h_{l}} \\
& \lesssim\left\|u-T_{h_{l}}(\lambda u)\right\|_{h_{l}}\left\|u^{h_{l}}-u\right\|_{h_{l}} \\
& \lesssim \delta_{h_{l}}^{2}(\lambda) .
\end{aligned}
$$

Substituting (59) and (61) into (60), (29) holds.

By (56) and triangle inequality, we have

$$
\begin{aligned}
\left\|u-u^{h_{l}}\right\|_{h_{l}, D}= & \left\|\left(T-T_{h_{l}}\right)\left(\lambda_{j, h_{l}} u^{h_{l}}\right)+R^{\prime}\right\|_{h_{l}, D} \\
\leq & \left\|\left(T-T_{h_{l}}\right)\left(\lambda^{h_{l}} u^{h_{l}}\right)\right\|_{h_{l}, D} \\
& +\left\|\left(T-T_{h_{l}}\right)\left(\lambda_{j, h_{l}}-\lambda^{h_{l}}\right) u^{h_{l}}+R^{\prime}\right\|_{h_{l}, D} \\
\equiv & \left\|\left(T-T_{h_{l}}\right)\left(\lambda^{h_{l}} u^{h_{l}}\right)\right\|_{h_{l}, D}+\|R\|_{h_{l}, D},
\end{aligned}
$$

where $R=\left(T-T_{h_{l}}\right)\left(\lambda_{j, h_{l}}-\lambda^{h_{l}}\right) u^{h_{l}}+R^{\prime}$.

By (57) and (29), we know that $\|R\|_{h_{l}, D} \lesssim \delta_{h_{l-1}}^{3}(\lambda)+\delta_{h_{l}}^{2}(\lambda)$; thus, (28) holds.

By calculation,

$$
\begin{aligned}
& \sum_{j=k}^{k+q-1} a_{h_{l}}\left(u^{h_{l}}, u_{j, h_{l}}\right) \lambda^{h_{l}} T_{h_{l}} u_{j, h_{l}} \\
& =\lambda^{h_{l}} T_{h_{l}}\left(\sum_{j=k}^{k+q-1} a_{h_{l}}\left(u^{h_{l}}, u_{j, h_{l}}\right) u_{j, h_{l}}\right) \\
& =\lambda^{h_{l}} T_{h_{l}} u^{*} .
\end{aligned}
$$

By the above formulae and (12), we deduce that

$$
\begin{aligned}
& \left\|u^{h_{l}}-T_{h_{l}}\left(\lambda^{h_{l}} u^{h_{l}}\right)\right\|_{h_{l}, D} \\
& =\| u^{h_{l}}-u^{*}+u^{*}-T_{h_{l}}\left(\lambda^{h_{l}} u^{*}\right) \\
& +T_{h_{l}}\left(\lambda^{h_{l}} u^{*}\right)-T_{h_{l}}\left(\lambda^{h_{l}} u^{h_{l}}\right) \|_{h_{l}, D} \\
& \lesssim\left\|u^{h_{l}}-u^{*}\right\|_{h_{l}, D} \\
& +\left\|\sum_{j=k}^{k+q-1}\left(\lambda_{j, h_{l}}-\lambda^{h_{l}}\right) a_{h_{l}}\left(u^{h_{l}}, u_{j, h_{l}}\right) T_{h_{l}} u_{j, h_{l}}\right\|_{h_{l}, D} \\
& +\left\|\lambda^{h_{l}} T_{h_{l}}\left(u^{*}-u^{h_{l}}\right)\right\|_{h_{l}, D} \\
& \lesssim\left\|u^{h_{l}}-u^{*}\right\|_{h_{l}, D}+\sum_{j=k}^{k+q-1}\left|\lambda_{j, h_{l}}-\lambda^{h_{l}}\right| \\
& \lesssim\left\|u^{h_{l}}-u^{*}\right\|_{h_{l}}+\delta_{h_{l}}^{2}(\lambda),
\end{aligned}
$$

which together with (49) leads to (30). This completes the proof.

Theorem 8. Let $\left(\lambda^{h_{l}}, u^{h_{l}}\right)$ be the kth approximate eigenpair of (1) obtained by Scheme 3, let $\lambda$ be the kth eigenvalue of (1), and let $H$ be properly small. Suppose that Condition 1 holds, then there exists $u \in M(\lambda)$ such that

$$
\begin{gathered}
\left\|u^{h_{l}}-u\right\|_{h_{l}} \leq C \delta_{h_{l}}(\lambda), \\
\left|\lambda^{h_{l}}-\lambda\right| \leq C \delta_{h_{l}}^{2}(\lambda), \quad l \geq 1 .
\end{gathered}
$$

Proof. The proof of (65) is completed by using induction. When $l=1$, by Lemma 6 , we know that Theorem 8 holds.

Suppose that Theorem 8 holds for $l-1$; that is,

$$
\begin{gathered}
\left\|u^{h_{l-1}}-u\right\|_{h_{l-1}} \leq C \delta_{h_{l-1}}(\lambda), \\
\left|\lambda^{h_{l-1}}-\lambda\right| \leq C \delta_{h_{l-1}}^{2}(\lambda),
\end{gathered}
$$

which together with the assumptions in Theorem 8 , we know that Theorem 7 holds. For $l$, by (29) and (59), we get (65). The proof is completed.

\section{A Posteriori Error Estimates for Multiscale Discretization Scheme}

Based on the work of $[14,26]$, in this section, we will discuss a posteriori error estimates of the C-R element approximation for Laplace eigenvalue problem.

Consider the boundary value problem corresponding to (2): find $w \in H_{0}^{1}(\Omega)$ such that

$$
a(w, v)=b(f, v), \quad \forall v \in H_{0}^{1}(\Omega),
$$

and its C-R element approximation: find $w_{h} \in V_{h}$ such that

$$
a_{h}\left(w_{h}, v\right)=b(f, v), \quad \forall v \in V_{h} .
$$


Let $\kappa^{+} \in \pi_{h}, \kappa^{-} \in \pi_{h}$ be two elements sharing one edge $e$. For any piecewise continuous function $\varphi$, we denote by $[[\varphi]]_{e}=\left.\left(\left.\varphi\right|_{\kappa^{+}}\right)\right|_{e}-\left.\left(\left.\varphi\right|_{\mathcal{K}^{-}}\right)\right|_{e}$ the jump of $\varphi$ across $e$.

Let $w_{h}$ be the solution of (68), $\widetilde{J}_{e, 9}$ be the jump of $\nabla w_{h}$ across $e$ along $\vartheta_{e}$, and $\widetilde{J}_{e, \tau}$ be the jump of $\nabla w_{h}$ across $e$ along $\tau_{e}$; let $\widetilde{R}_{\kappa}\left(w_{h}\right)$ be element residual; that is,

$$
\begin{aligned}
& \widetilde{R}_{\kappa}\left(w_{h}\right)=f+\Delta w_{h} \quad \kappa \in \pi_{h}, \\
& \widetilde{J}_{e, \vartheta}\left(w_{h}\right)= \begin{cases}{\left[\left[\nabla w_{h}\right]\right]_{e} \cdot \vartheta_{e},} & e \in \varepsilon(\Omega), \\
0, & e \in \varepsilon(\partial \Omega),\end{cases} \\
& \widetilde{J}_{e, \tau}\left(w_{h}\right)= \begin{cases}{\left[\left[\nabla w_{h}\right]\right]_{e} \cdot \tau_{e},} & e \in \varepsilon(\Omega), \\
-\sqrt{2} \nabla w_{h} \cdot \tau_{e}, & e \in \varepsilon(\partial \Omega) .\end{cases}
\end{aligned}
$$

For $\kappa \in \pi_{h}$, define the residual on the element $\kappa$ as

$$
\begin{aligned}
\widetilde{\eta}_{h}\left(w_{h}, \kappa\right)=\left(h_{\kappa}^{2}\left\|\widetilde{R}_{\kappa}\left(w_{h}\right)\right\|_{0, \kappa}^{2}\right. \\
\left.\quad+\frac{1}{2} \sum_{e \in \partial \kappa} h_{e}\left(\left\|\widetilde{J}_{e, \tau}\left(w_{h}\right)\right\|_{0, e}^{2}+\left\|\widetilde{J}_{e, \vartheta}\left(w_{h}\right)\right\|_{0, e}^{2}\right)\right)^{1 / 2},
\end{aligned}
$$

and thus, for $G \subset \Omega$, the residual sum on $G$ is given by

$$
\tilde{\eta}_{h}\left(w_{h}, G\right)=\left(\sum_{\kappa \in \pi_{h}, \kappa \subset G} \widetilde{\eta}_{h}^{2}\left(w_{h}, \kappa\right)\right)^{1 / 2} .
$$

For $f \in L^{2}(\Omega)$, define the date oscillation by

$$
\operatorname{osc}\left(f, \pi_{h}\right)=\left(\sum_{\kappa \in \pi_{h}} h_{\kappa}^{2}\left\|f-f_{h}\right\|_{0, \kappa}^{2}\right)^{1 / 2},
$$

where $f_{h}$ stands for a piecewise polynomial approximation of $f$ over $\pi_{h}$.

For the boundary value problem (67), Carstensen and $\mathrm{Hu}$ [27] have proved the following a posteriori error estimates :

$$
\left\|w-w_{h}\right\|_{h, \Omega} \leq \widetilde{C}_{1}\left(\widetilde{\eta}_{h}\left(w_{h}, \Omega\right)+\operatorname{osc}\left(f, \pi_{h}\right)\right),
$$

where constant $\widetilde{C}_{1}$ is only dependent on minimum angle of $\pi_{h}$, and if the right-hand side $f$ of (67) is a piecewise linear polynomial over $\pi_{h}$, then

$$
\widetilde{C}_{2} \widetilde{\eta}_{h}\left(w_{h}, \Omega\right) \leq\left\|w-w_{h}\right\|_{h, \Omega} .
$$

Selecting $f=\lambda^{h_{l}} u^{h_{l}}$ in (67) and (68), then the generalized solution and the nonconforming finite element solution are $w=T\left(\lambda^{h_{l}} u^{h_{l}}\right)$ and $w_{h}=T_{h_{l}}\left(\lambda^{h_{l}} u^{h_{l}}\right)$, respectively, and the a posteriori error indicator of $w_{h}$ is $\widetilde{\eta}_{h}\left(w_{h}, \Omega\right)$, which is defined by (71).
Define the element residual $R_{\kappa}\left(u^{h_{l}}\right)$ and the jump residual $J_{e, 9}\left(u^{h_{l}}\right)$ and $J_{e, \tau}\left(u^{h_{l}}\right)$ for $u^{h_{l}}$ as follows:

$$
\begin{aligned}
R_{\kappa}\left(u^{h_{l}}\right) & =\lambda^{h_{l}} u^{h_{l}}+\Delta u^{h_{l}} \quad \kappa \in \pi_{h_{l},} \\
J_{e, 9}\left(u^{h_{l}}\right) & = \begin{cases}{\left[\left[\nabla u^{h_{l}}\right]\right]_{e} \cdot \vartheta_{e},} & e \in \varepsilon(\Omega), \\
0, & e \in \varepsilon(\partial \Omega),\end{cases} \\
J_{e, \tau}\left(u^{h_{l}}\right) & = \begin{cases}{\left[\left[\nabla u^{h_{l}}\right]\right]_{e} \cdot \tau_{e},} & e \in \varepsilon(\Omega), \\
-\sqrt{2} \nabla u^{h_{l}} \cdot \tau_{e}, & e \in \varepsilon(\partial \Omega) .\end{cases}
\end{aligned}
$$

For $\kappa \in \pi_{h_{l}}$, define the residual on the element $\kappa$ as

$$
\begin{aligned}
\eta_{h_{l}}\left(u^{h_{l}}, \kappa\right)= & \left(h_{\kappa}^{2}\left\|R_{\kappa}\left(u^{h_{l}}\right)\right\|_{0, \kappa}^{2}\right. \\
& \left.+\frac{1}{2} \sum_{e \in \partial \kappa} h_{e}\left(\left\|J_{e, \tau}\left(u^{h_{l}}\right)\right\|_{0, e}^{2}+\left\|J_{e, \vartheta}\left(u^{h_{l}}\right)\right\|_{0, e}^{2}\right)\right)^{1 / 2} .
\end{aligned}
$$

For $G \subset \Omega$, define the residual sum on $G$ as

$$
\eta_{h_{l}}\left(u^{h_{l}}, G\right)=\left(\sum_{\kappa \in \pi_{h_{l}}, \kappa \subset G} \eta_{h_{l}}^{2}\left(u^{h_{l}}, \kappa\right)\right)^{1 / 2} .
$$

Theorem 9. Suppose that the conditions in Theorem 7 hold and $V_{h_{l}}$ is a finite element space consisting of piecewise linear polynomials, then there exists a positive constant $\delta$ which is independent of mesh parameter, such that

$$
\begin{gathered}
\left\|u-u^{h_{l}}\right\|_{h_{l}, \Omega} \leq\left(\widetilde{C}_{1}+\delta\right) \eta_{h_{l}}\left(u^{h_{l}}, \Omega\right), \\
\left(\widetilde{C}_{2}+\delta\right) \eta_{h_{l}}\left(u^{h_{l}}, \Omega\right) \leq\left\|u-u^{h_{l}}\right\|_{h_{l}, \Omega}, \\
\left|\lambda-\lambda^{h_{l}}\right| \leq \eta_{h_{l}}^{2}\left(u^{h_{l}}, \Omega\right) .
\end{gathered}
$$

Proof. Let $w_{h_{l}}=T_{h_{l}} \lambda^{h_{l}} u^{h_{l}}$, and by calculation

$$
\begin{aligned}
\widetilde{\eta}_{h_{l}}\left(w_{h_{l}}, \Omega\right)= & \left(\sum_{\kappa \in \pi_{h_{l}, \kappa \subset \Omega}} \widetilde{\eta}_{h_{l}}^{2}\left(w_{h_{l}}, \kappa\right)\right)^{1 / 2} \\
= & \left(\sum_{\kappa \in \pi_{h_{l}, \kappa \subset \Omega}} \eta_{h_{l}}^{2}\left(u^{h_{l}}, \kappa\right)\right)^{1 / 2} \\
& +\left(\sum_{\kappa \in \pi_{h_{l}, \kappa \subset \Omega}} \widetilde{\eta}_{h_{l}}^{2}\left(w_{h_{l}}, \kappa\right)\right)^{1 / 2} \\
& -\left(\sum_{\kappa \in \pi_{h_{l}, \kappa \subset \Omega}} \eta_{h_{l}}^{2}\left(u^{h_{l}}, \kappa\right)\right)^{1 / 2} \\
\equiv & \eta_{h_{l}}\left(u^{h_{l}}, \Omega\right)+R_{2} .
\end{aligned}
$$


By triangle inequality, we have

$$
\begin{aligned}
\left|R_{2}\right|= & \mid\left(\sum_{\kappa \in \pi_{h_{l}, \kappa \subset \Omega}} \tilde{\eta}_{h_{l}}^{2}\left(w_{h_{l}}, \kappa\right)\right)^{1 / 2} \\
& -\left(\sum_{\kappa \in \pi_{h_{l}}, \kappa \subset \Omega} \eta_{h_{l}}^{2}\left(u^{h_{l}}, \kappa\right)\right)^{1 / 2} \mid \\
\leq & \left(\sum_{\kappa \in \pi_{h_{l}, \kappa \subset \Omega}}\left(\tilde{\eta}_{h_{l}}\left(w_{h_{l}}, \kappa\right)-\eta_{h_{l}}\left(u^{h_{l}}, \kappa\right)\right)^{2}\right)^{1 / 2} .
\end{aligned}
$$

From triangle inequality, (69)-(70), and (75)-(76), we deduce that

$$
\begin{gathered}
\left|\widetilde{\eta}_{h_{l}}\left(w_{h_{l}}, \kappa\right)-\eta_{h_{l}}\left(u^{h_{l}}, \kappa\right)\right| \\
=\mid\left(h_{\kappa}^{2}\left\|\widetilde{R}_{\kappa}\left(w_{h_{l}}\right)\right\|_{0, \kappa}^{2}\right. \\
\left.+\frac{1}{2} \sum_{e \in \partial \kappa} h_{e}\left(\left\|\widetilde{J}_{e, \tau}\left(w_{h_{l}}\right)\right\|_{0, e}^{2}+\left\|\widetilde{J}_{e, 9}\left(w_{h_{l}}\right)\right\|_{0, e}^{2}\right)\right)^{1 / 2} \\
-\left(h_{\kappa}^{2}\left\|R_{\kappa}\left(u^{h_{l}}\right)\right\|_{0, \kappa}^{2}\right. \\
\left.+\frac{1}{2} \sum_{e \in \partial \kappa} h_{e}\left(\left\|J_{e, \tau}\left(u^{h_{l}}\right)\right\|_{0, e}^{2}+\left\|J_{e, \vartheta}\left(u^{h_{l}}\right)\right\|_{0, e}^{2}\right)\right)^{1 / 2} \mid \\
\leq\left(h_{\kappa}^{2}\left\|\widetilde{R}_{\kappa}\left(w_{h_{l}}\right)-R_{\kappa}\left(u^{h_{l}}\right)\right\|_{0, \kappa}^{2}\left(w_{h_{l}}-u^{h_{l}}\right) \|_{0, \kappa}^{2}\right. \\
+\frac{1}{2} \sum_{e \in \partial \kappa} \sum_{e}\left(\left\|\tilde{J}_{e, \tau}\left(w_{h_{l}}\right)-J_{e, \tau}\left(u^{h_{l}}\right)\right\|_{0, e}^{2}\right. \\
\left.+\|\left[\tilde{J}_{e, \vartheta}\left(w_{h_{l}}\right)-J_{l, 9}\left(u^{h_{l}}\right) \|_{0, e}^{2}\right)\right)^{1 / 2}
\end{gathered}
$$

It is obvious that $\left\|\Delta\left(w_{h_{l}}-u^{h_{l}}\right)\right\|_{0, \kappa}^{2}=0$, and, by the trace theorem (see e.g., [28]) and the inverse estimates, we get

$$
\begin{gathered}
\frac{1}{2} \sum_{e \in \partial \kappa} h_{e}\left(\left\|\left[\left[\nabla\left(w_{h_{l}}-u^{h_{l}}\right)\right]\right]_{e} \cdot \vartheta_{e}\right\|_{0, e}^{2}\right. \\
\left.+\left\|\left[\left[\nabla\left(w_{h_{l}}-u^{h_{l}}\right)\right]\right]_{e} \cdot \tau_{e}\right\|_{0, e}^{2}\right) \\
\leq h_{\kappa}\left(h_{\kappa}^{-1}\left\|\nabla\left(w_{h_{l}}-u^{h_{l}}\right)\right\|_{0, \omega_{\kappa}}^{2}\right. \\
\left.+h_{\kappa}\left|\nabla\left(w_{h_{l}}-u^{h_{l}}\right)\right|_{1, \omega_{\kappa}}^{2}\right) \\
\leq\left\|w_{h_{l}}-u^{h_{l}}\right\|_{h_{l}, \omega_{\kappa}}^{2} \cdot
\end{gathered}
$$

Thus,

$$
\begin{aligned}
\left|\widetilde{\eta}_{h_{l}}\left(w_{h_{l}}, \kappa\right)-\eta_{h_{l}}\left(u^{h_{l}}, \kappa\right)\right| & \lesssim\left\|T_{h_{l}}\left(\lambda^{h_{l}} u^{h_{l}}\right)-u^{h_{l}}\right\|_{h_{l}, \kappa} \\
& =\left\|w_{h_{l}}-u^{h_{l}}\right\|_{h_{l}, \omega_{\kappa}} .
\end{aligned}
$$

Combining (82), (85), and (30), we get

$$
\left|R_{2}\right| \lesssim\left\|w_{h_{l}}-u^{h_{l}}\right\|_{h_{l}} \lesssim \delta_{h_{l-1}}^{3}(\lambda)+\delta_{h_{l}}^{2}(\lambda) .
$$

Hence, from Condition 1 , we know that $R_{2}$ is a small quantity of higher order than $\tilde{\eta}_{h_{l}}\left(w_{h_{l}}, \Omega\right)$. Using (81), we obtain that $R_{2}$ is also a small quantity of higher order than $\eta_{h_{l}}\left(u^{h_{l}}, \Omega\right)$.

Therefore, by (28), (73), (81), and (86), we have

$$
\begin{aligned}
\| u- & u^{h_{l}} \|_{h_{l}, \Omega} \\
= & \left\|\left(T-T_{h_{l}}\right)\left(\lambda^{h_{l}} u^{h_{l}}\right)\right\|_{h_{l}, \Omega}+\|R\|_{h_{l}, \Omega} \\
\leq & \widetilde{C}_{1} \widetilde{\eta}_{h_{l}}\left(T_{h_{l}}\left(\lambda^{h_{l}} u^{h_{l}}\right), \Omega\right)+\|R\|_{h_{l}, \Omega} \\
\leq & \widetilde{C}_{1} \eta_{h_{l}}\left(u^{h_{l}}, \Omega\right) \\
& +\widetilde{C}_{1}\left(\widetilde{\eta}_{h_{l}}\left(T_{h_{l}}\left(\lambda^{h_{l}} u^{h_{l}}\right), \Omega\right)-\eta_{h_{l}}\left(u^{h_{l}}, \Omega\right)\right) \\
& +\|R\|_{h_{l}, \Omega} \\
\leq & \widetilde{C}_{1} \eta_{h_{l}}\left(u^{h_{l}}, \Omega\right)+\widetilde{C}_{1}\left\|R_{2}\right\|_{h_{l}, \Omega}+\|R\|_{h_{l}, \Omega} \\
\leq & \left(\widetilde{C}_{1}+\delta\right) \eta_{h_{l}}\left(u^{h_{l}}, \Omega\right),
\end{aligned}
$$


Similarly, by (28) and (74), we get

$$
\begin{aligned}
\| u- & u^{h_{l}} \|_{h_{l}, \Omega} \\
= & \left\|\left(T-T_{h_{l}}\right)\left(\lambda^{h_{l}} u^{h_{l}}\right)\right\|_{h_{l}, \Omega}+\|R\|_{h_{l}, \Omega} \\
\geq & \widetilde{C}_{2} \widetilde{\eta}_{h_{l}}\left(T_{h_{l}}\left(\lambda^{h_{l}} u^{h_{l}}\right), \Omega\right)+\|R\|_{h_{l}, \Omega} \\
\geq & \widetilde{C}_{2} \eta_{h_{l}}\left(u^{h_{l}}, \Omega\right) \\
& +\widetilde{C}_{2}\left(\widetilde{\eta}_{h_{l}}\left(T_{h_{l}}\left(\lambda^{h_{l}} u^{h_{l}}\right), \Omega\right)-\eta_{h_{l}}\left(u^{h_{l}}, \Omega\right)\right) \\
& +\|R\|_{h_{l}, \Omega} \\
\geq & \widetilde{C}_{2} \eta_{h_{l}}\left(u^{h_{l}}, \Omega\right)+\widetilde{C}_{2}\left\|R_{2}\right\|_{h_{l}, \Omega}+\|R\|_{h_{l}, \Omega} \\
\geq & \left(\widetilde{C}_{2}+\delta\right) \eta_{h_{l}}\left(u^{h_{l}}, \Omega\right),
\end{aligned}
$$

and thus (79) holds.

By (61) and (28), we get

$$
\begin{aligned}
E_{h_{l}}\left(u, u^{h_{l}}\right) & \lesssim\left\|u-T_{h_{l}}(\lambda u)\right\|_{h_{l}}\left\|u^{h_{l}}-u\right\|_{h_{l}} \\
& \lesssim\left\|u^{h_{l}}-u\right\|_{h_{l}}^{2}
\end{aligned}
$$

and, by substituting the above relation into (60), we obtain

$$
\left|\lambda^{h_{l}}-\lambda\right| \lesssim\left\|u^{h_{l}}-u\right\|_{h_{l}}^{2}
$$

which together with (78) yields (80). This completes the proof.

\section{Adaptive Finite Element Algorithm Based on Multiscale Discretizations}

As we know, The following Algorithm 10 is fundamental and important; see $[14,16]$ for its detailed theoretical results.

Algorithm 10. Choose parameter $0<\theta<1$.

Step 1. Pick any initial mesh $\pi_{h_{0}}$ with mesh size $h_{0}$.

Step 2. Solve (5) on $\pi_{h_{0}}$ for discrete solution $\left(\lambda_{h_{0}}, u_{h_{0}}\right)$.

Step $3 . l \Leftarrow 0$.

Step 4. Compute the local indicators $\eta_{h_{l}}\left(u_{h_{l}}, \kappa\right)$.

Step 5. Construct $\hat{\pi}_{h_{l}} \subset \pi_{h_{l}}$ by Marking Strategy $E$ and parameter $\theta$.

Step 6. Refine $\pi_{h_{l}}$ to get a new mesh $\pi_{h_{l+1}}$.

Step 7. Solve (5) on $\pi_{h_{l+1}}$ for discrete solution $\left(\lambda_{h_{l+1}}, u_{h_{l+1}}\right)$.

Step $8 . l \Leftarrow l+1$, and go to Step 4 .
Marking Strategy E. Give parameter $0<\theta<1$.

Step 1. Construct a minimal subset $\widehat{\pi}_{h_{l}}$ of $\pi_{h_{l}}$ by selecting some elements in $\pi_{h_{l}}$ such that

$$
\sum_{\kappa \in \hat{\pi}_{h_{l}}} \eta_{h_{l}}^{2}\left(u_{h_{l}}, \kappa\right) \geq \theta \eta_{h_{l}}^{2}\left(u_{h_{l}}, \Omega\right) .
$$

Step 2. Mark all the elements $\widehat{\pi}_{h_{l}}$.

$\eta_{h_{l}}\left(u_{h_{l}}, \kappa\right)$ and $\eta_{h_{l}}\left(u_{h_{l}}, \Omega\right)$ are defined as (76) and (77), respectively, with $u^{h_{l}}$ and $\lambda^{h_{l}}$ replaced by $u_{h_{l}}$ and $\lambda_{h_{l}}$.

We have the following adaptive algorithm on the basis of Scheme 3.

Algorithm 11. Choose parameter $0<\theta<1$.

Step 1. Pick any initial mesh $\pi_{h_{0}}$ with mesh size $h_{0}$.

Step 2. Solve (5) on $\pi_{h_{0}}$ for discrete solution $\left(\lambda^{h_{0}}, u^{h_{0}}\right)$.

Step 3. $l \Leftarrow 0, \lambda_{0} \Leftarrow \lambda^{h_{0}}$.

Step 4. Compute the local indicators $\eta_{h_{l}}\left(u^{h_{l}}, \kappa\right)$.

Step 5. Construct $\hat{\pi}_{h_{l}} \subset \pi_{h_{l}}$ by Marking Strategy $E$ and parameter $\theta$.

Step 6. Refine $\pi_{h_{l}}$ to get a new mesh $\pi_{h_{l+1}}$

Step 7. Find $u^{\prime} \in V_{h_{l+1}}$ such that

$$
a_{h_{l+1}}\left(u^{\prime}, \psi\right)-\lambda_{0} b\left(u^{\prime}, \psi\right)=b\left(u^{h_{l}}, \psi\right), \quad \forall \psi \in V_{h_{l+1}} .
$$

Set $u^{h_{l+1}}=u^{\prime} /\left\|u^{\prime}\right\|_{h_{l+1}}$ and compute the Rayleigh quotient

$$
\lambda^{h_{l+1}}=\frac{a_{h_{l+1}}\left(u^{h_{l+1}}, u^{h_{l+1}}\right)}{b\left(u^{h_{l+1}}, u^{h_{l+1}}\right)} .
$$

Step 8. $\lambda_{0} \Leftarrow \lambda^{h_{l+1}}, l \Leftarrow l+1$ and go to Step 4 .

Marking Strategy $E$ in Algorithm 11 will be the same as that in Algorithm 10, except for replacing $u_{h_{l}}$ with $u^{h_{l}}$.

Note that when $\left|\lambda_{0}-\lambda\right|$ is too small, (92) is an almost singular linear equation. Although it has no difficulty in solving (92) numerically (see Lecture 27.4 in [29]), one would like to think of selecting a proper integer $l_{0} \geq 0$. When $l \geq l_{0}$, set $\lambda^{h_{l}}=\lambda^{h_{l_{0}}}$ in (92). So, we can establish the following algorithm (see e.g., Scheme 3.2 in [24]).

Algorithm 12. Choose parameter $0<\theta<1$.

Step 1-Step 7. Execute Step 1-Step 7 of Algorithm 11.

Step 8. If $l<l_{0}, \lambda_{0} \Leftarrow \lambda^{h_{l+1}}, l \Leftarrow l+1$, go to Step 4; else $l \Leftarrow l+1$, go to Step 4 .

Marking Strategy E in Algorithm 12 will be the same as that in Algorithm 11. 


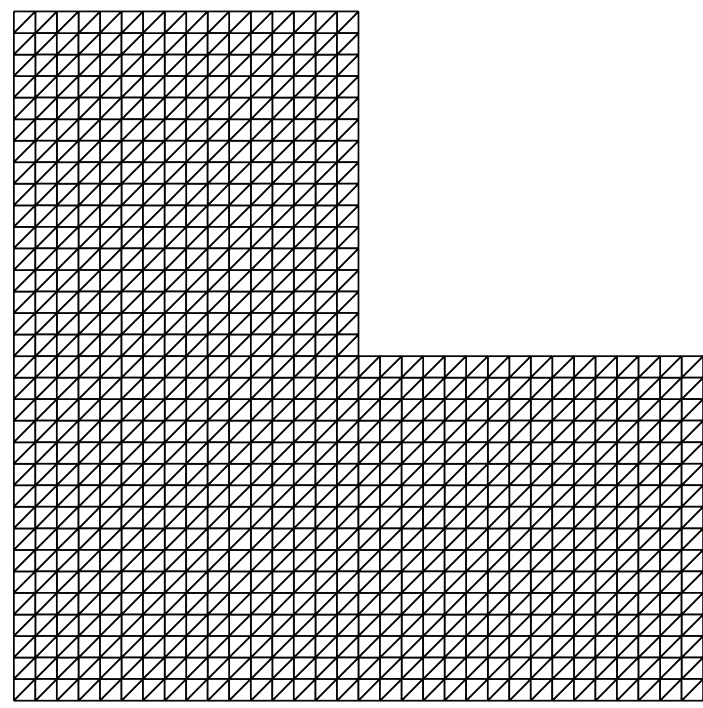

(a)

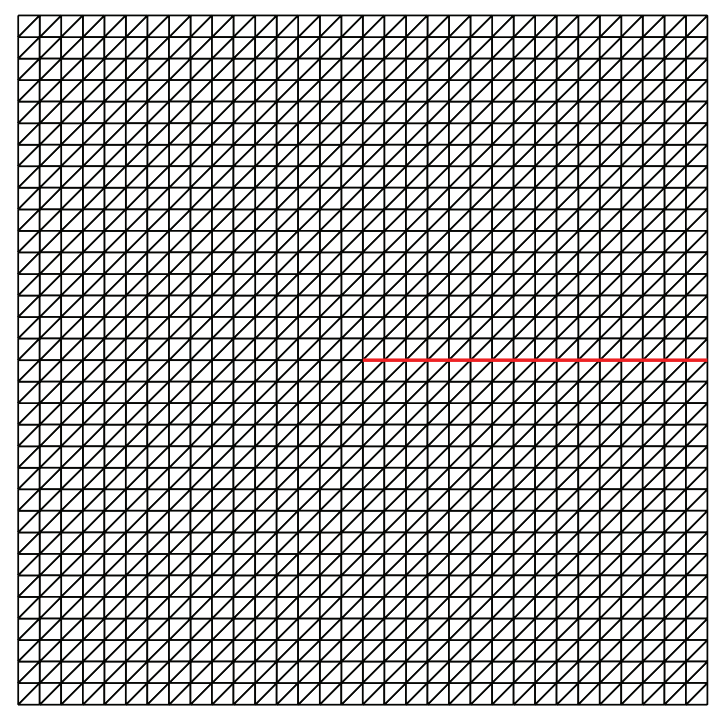

(b)

FIGURE 1: The initial mesh $H=\sqrt{2} / 16$.

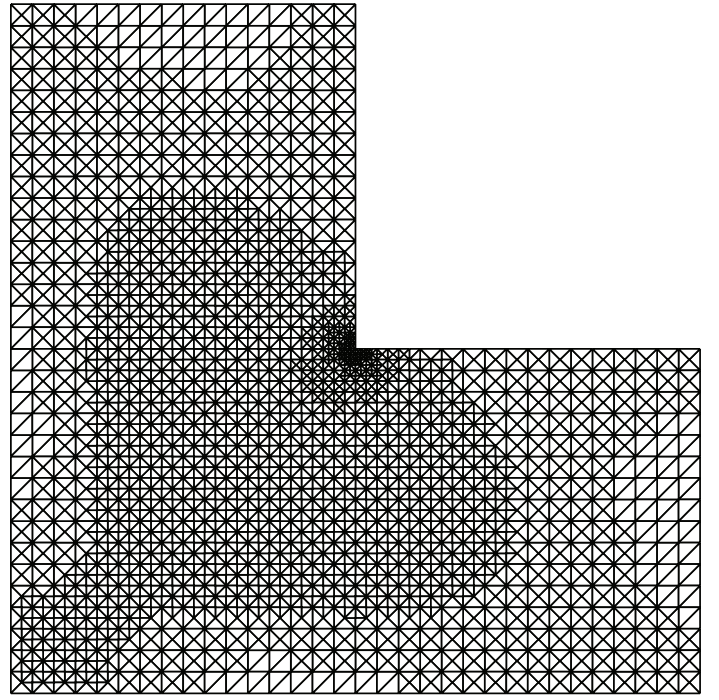

(a)

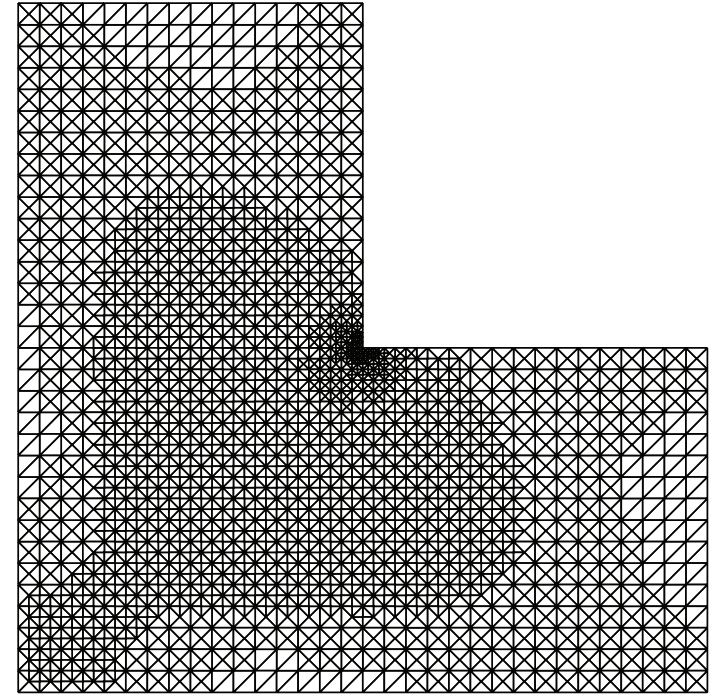

(b)

FIgURE 2: The adaptive meshes of 1st eigenvalue after 5th iteration by Algorithm 10 (a) and Algorithm 11 (b).

\section{Numerical Experiments}

In this section, we will report two numerical examples for Algorithms 10 and 11 to illustrate the theoretical results in this paper. We use MATLAB 2012 to solve Examples 1 and 2. Our program is compiled under the package of Chen. We take $\theta=0.5$ in two Algorithms.

For reading convenience, we use the following notations in our tables.

\section{$l^{*}:$ The $l^{*}$ th iteration of Algorithm 10}

$\lambda_{k, h_{l^{*}}}:$ The $k$ th approximate eigenvalue derived from the $l^{*}$ th iteration obtained by Algorithm 10 $\operatorname{dof}_{k, l^{*}}:$ The degrees of freedom of the $l^{*}$ th iteration for computing $\lambda_{k, h_{l^{*}}}$

$\mathrm{CPU}_{k, l^{*}}(s)$ : The total CPU time(s) for computing $\lambda_{k, h_{l} *}$

$\left|\lambda_{k, h_{l^{*}}}-\lambda_{k}\right|:$ the error of $k$ th approximate eigenvalue $\lambda_{k, h_{l^{*}}}$

l: The $l$ th iteration of Algorithm 11

$\lambda_{k}^{h_{l}}$ :The $k$ th approximate eigenvalue derived from the $l$ th iteration obtained by Algorithm 11

$\operatorname{dof}_{k, l}$ : The degrees of freedom of the $l$ th iteration for computing $\lambda_{k}^{h_{l}}$ 


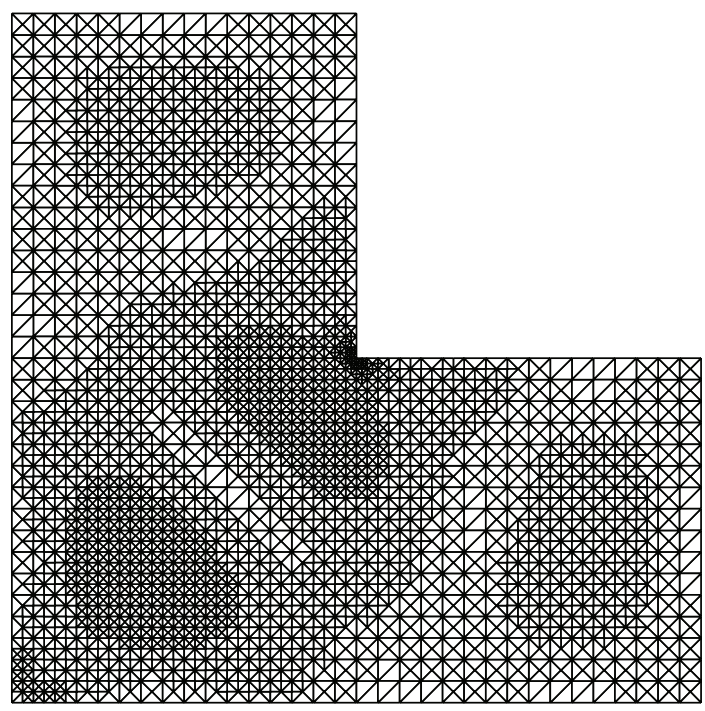

(a)

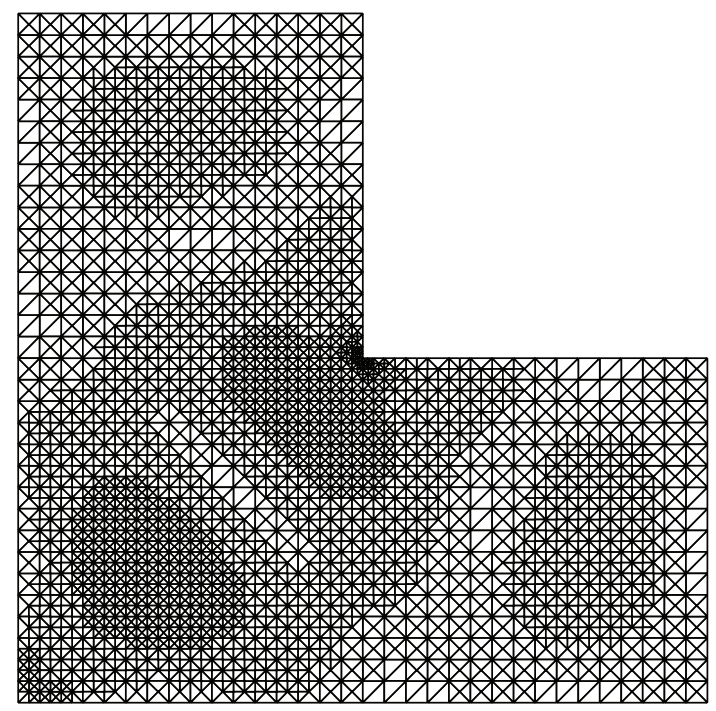

(b)

Figure 3: The adaptive meshes of 5th eigenvalue after 5th iteration by Algorithm 10 (a) and Algorithm 11 (b).

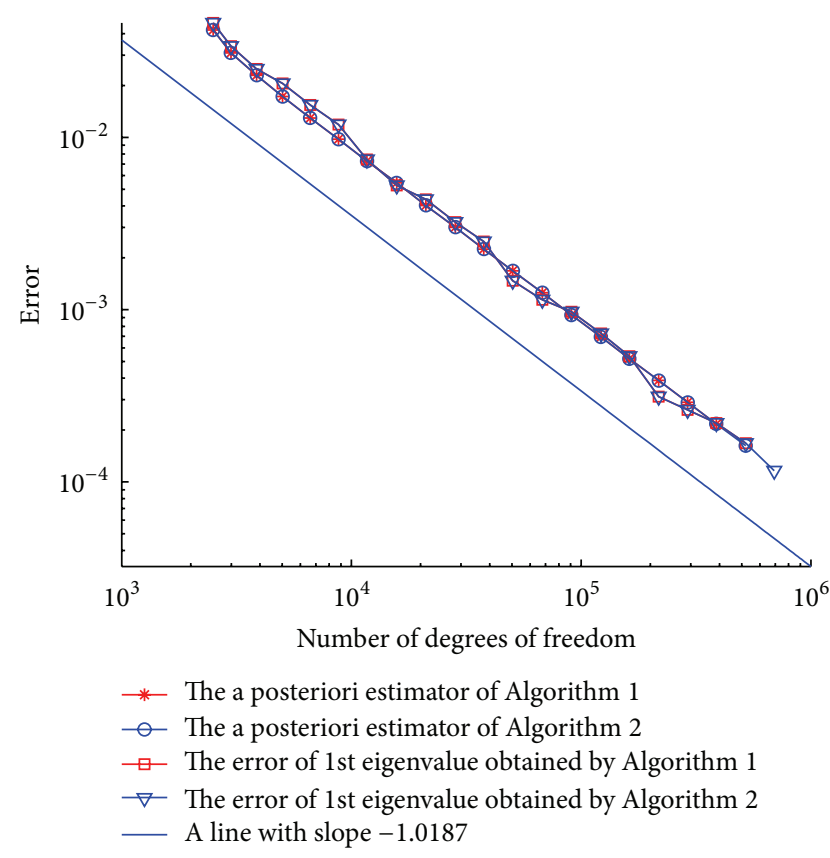

(a)

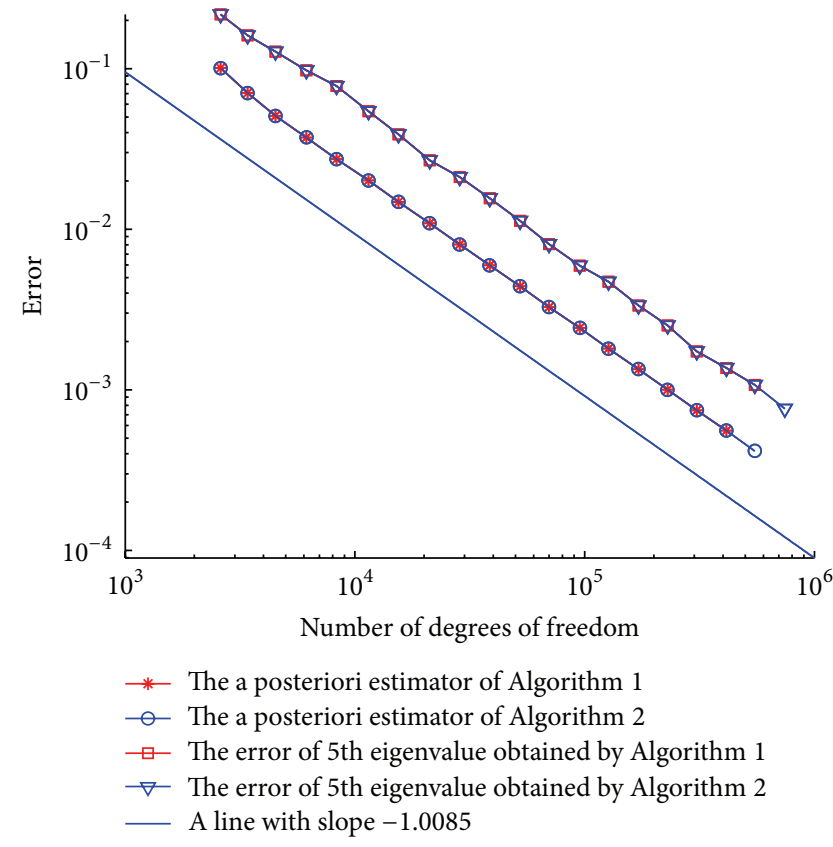

(b)

FIGURE 4: The error curves of two algorithms on $L$-shaped domain.

$$
\begin{aligned}
& \mathrm{CPU}_{k, l}(s) \text { : The total CPU time(s) for computing } \lambda_{k}^{h_{l}} \\
& \left|\lambda_{k}^{h_{l}}-\lambda_{k}\right| \text { : The error of } k \text { th approximate eigenvalue } \lambda_{k}^{h_{l}} \text {. }
\end{aligned}
$$

Example 1. We use Algorithms 10 and 11 to compute the approximate eigenvalues of (1) on the $L$-shaped domain $\Omega=$ $((0,2) \times(0,2)) \backslash([1,2] \times[1,2])($ see Figure $1(\mathrm{a}))$.

The first and fifth eigenvalues of $(1)$ are $\lambda_{1} \approx 9.639723844$ and $\lambda_{5} \approx 31.912636$ on this domain, respectively. The associated numerical results are presented in Table 1 and
Figures 1(a), 2, 3, and 4. Figure 1(a) gives the uniform initial mesh with $H=\sqrt{2} / 16$. Figures 2 and 3 show the adaptive meshes of the first and fifth eigenvalues after the fifth iteration by two algorithms, respectively. It is indicated in Figure 4 that the error curves of the first and fifth approximate eigenvalues and the curves of the associated a posteriori error estimators obtained by Algorithms 10 and 11 are approximately parallel to the line with slope -1 , respectively; this coincides with our theory in Section 4. 
TABLE 1: The 1st and 5th eigenvalues obtained by two algorithms on $L$-shaped domain with $H=\sqrt{2} / 16$.

\begin{tabular}{|c|c|c|c|c|c|c|c|c|}
\hline$k$ & $l^{*}$ & $\operatorname{dof}_{k, l^{*}}$ & $\lambda_{k, h_{l^{*}}}$ & $\mathrm{CPU}_{k, l^{*}}(\mathrm{~s})$ & $l$ & $\operatorname{dof}_{k, l}$ & $\lambda_{k}^{h_{l}}$ & $\mathrm{CPU}_{k, l}(\mathrm{~s})$ \\
\hline 1 & 5 & 6615 & 9.624308 & 0.43 & 5 & 6615 & 9.624308 & 0.26 \\
\hline 1 & 10 & 28350 & 9.636503 & 1.83 & 10 & 28350 & 9.636503 & 0.99 \\
\hline 1 & 15 & 122122 & 9.638997 & 9.27 & 15 & 122122 & 9.638997 & 5.17 \\
\hline 1 & 19 & 387527 & 9.639505 & 35.6 & 19 & 387527 & 9.639505 & 19.3 \\
\hline 1 & 20 & 520999 & 9.639556 & 52.0 & 20 & 520999 & 9.639556 & 26.6 \\
\hline 1 & 21 & - & - & - & 21 & 692323 & 9.639608 & 38.2 \\
\hline 5 & 5 & 8319 & 31.83478 & 0.62 & 5 & 8319 & 31.83478 & 0.35 \\
\hline 5 & 10 & 38601 & 31.89705 & 3.07 & 10 & 38601 & 31.89705 & 1.75 \\
\hline 5 & 15 & 171591 & 31.90928 & 17.1 & 15 & 171591 & 31.90928 & 9.79 \\
\hline 5 & 18 & 414327 & 31.91127 & 47.9 & 18 & 414327 & 31.91127 & 26.7 \\
\hline 5 & 19 & 550331 & 31.91156 & 68.6 & 19 & 550331 & 31.91156 & 37.0 \\
\hline 5 & 20 & - & - & - & 20 & 743563 & 31.91187 & 53.0 \\
\hline
\end{tabular}

TABLE 2: The 1st and 6th eigenvalues obtained by two algorithms on slit domain with $H=\sqrt{2} / 16$.

\begin{tabular}{|c|c|c|c|c|c|c|c|c|}
\hline$k$ & $l^{*}$ & $\operatorname{dof}_{k, l^{*}}$ & $\lambda_{k, h_{l^{*}}}$ & $\mathrm{CPU}_{k, l^{*}}(\mathrm{~s})$ & $l$ & $\operatorname{dof}_{k, l}$ & $\lambda_{k}^{h_{l}}$ & $\mathrm{CPU}_{k, l}(\mathrm{~s})$ \\
\hline 1 & 5 & 5229 & 8.329697 & 0.43 & 5 & 5229 & 8.329697 & 0.25 \\
\hline 1 & 10 & 17488 & 8.361012 & 1.33 & 10 & 17491 & 8.361012 & 0.79 \\
\hline 1 & 15 & 64863 & 8.368891 & 5.14 & 15 & 64863 & 8.368891 & 2.90 \\
\hline 1 & 20 & 249713 & 8.370756 & 23.4 & 20 & 249713 & 8.370756 & 12.9 \\
\hline 1 & 23 & 569429 & 8.371082 & 61.5 & 23 & 569429 & 8.371082 & 31.2 \\
\hline 1 & 24 & - & - & - & 24 & 748279 & 8.371148 & 44.0 \\
\hline 6 & 5 & 8733 & 30.39428 & 0.69 & 5 & 8721 & 30.39422 & 0.41 \\
\hline 6 & 10 & 35253 & 30.50367 & 3.21 & 10 & 35212 & 30.50363 & 1.68 \\
\hline 6 & 15 & 141116 & 30.52867 & 16.2 & 15 & 140930 & 30.52866 & 8.20 \\
\hline 6 & 19 & 426746 & 30.53371 & 58.8 & 19 & 426194 & 30.53371 & 28.9 \\
\hline 6 & 20 & 566328 & 30.53432 & 82.6 & 20 & 565526 & 30.53431 & 39.1 \\
\hline 6 & 21 & - & - & - & 21 & 743261 & 30.53464 & 54.9 \\
\hline
\end{tabular}

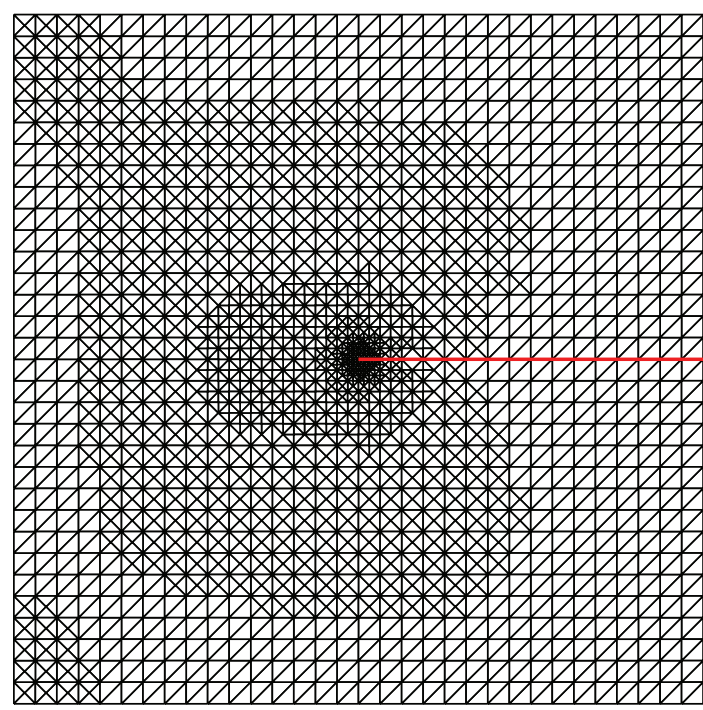

(a)

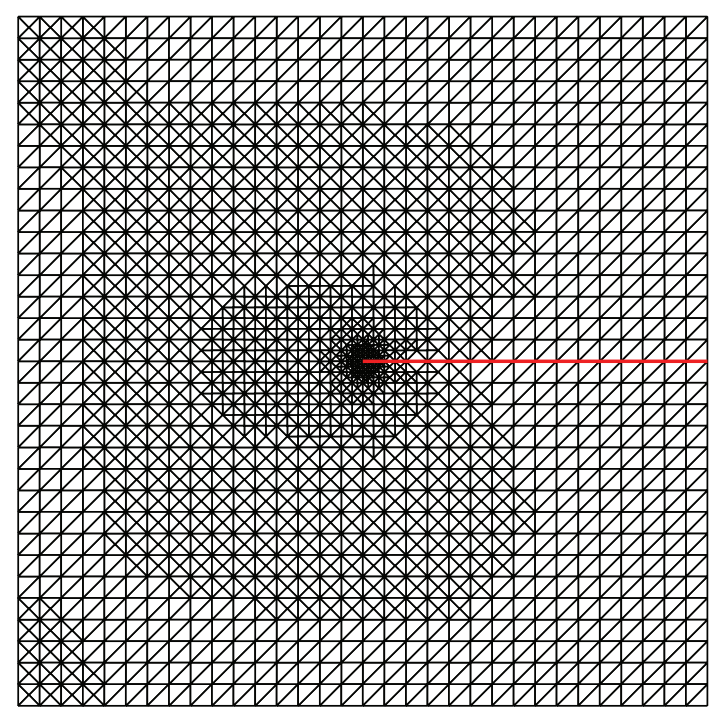

(b)

FIgURE 5: The adaptive meshes of 1st eigenvalue after 5th iteration by Algorithm 10 (a) and Algorithm 11 (b). 


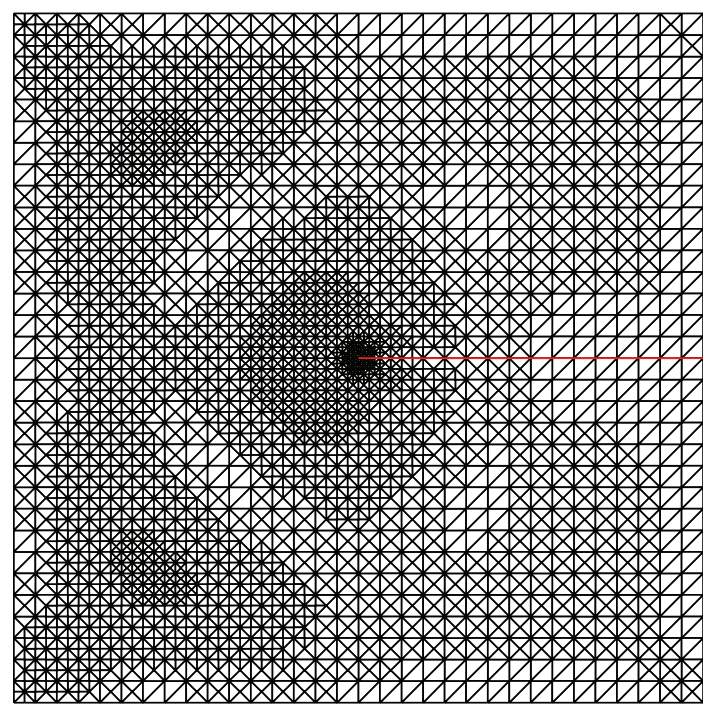

(a)

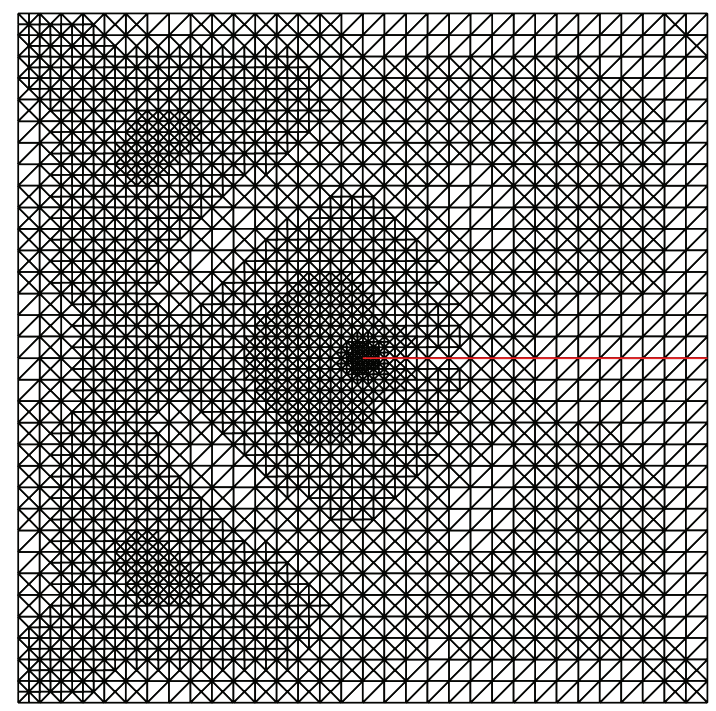

(b)

FIgURE 6: The adaptive meshes of 6th eigenvalue after 5th iteration by Algorithm 10 (a) and Algorithm 11 (b).

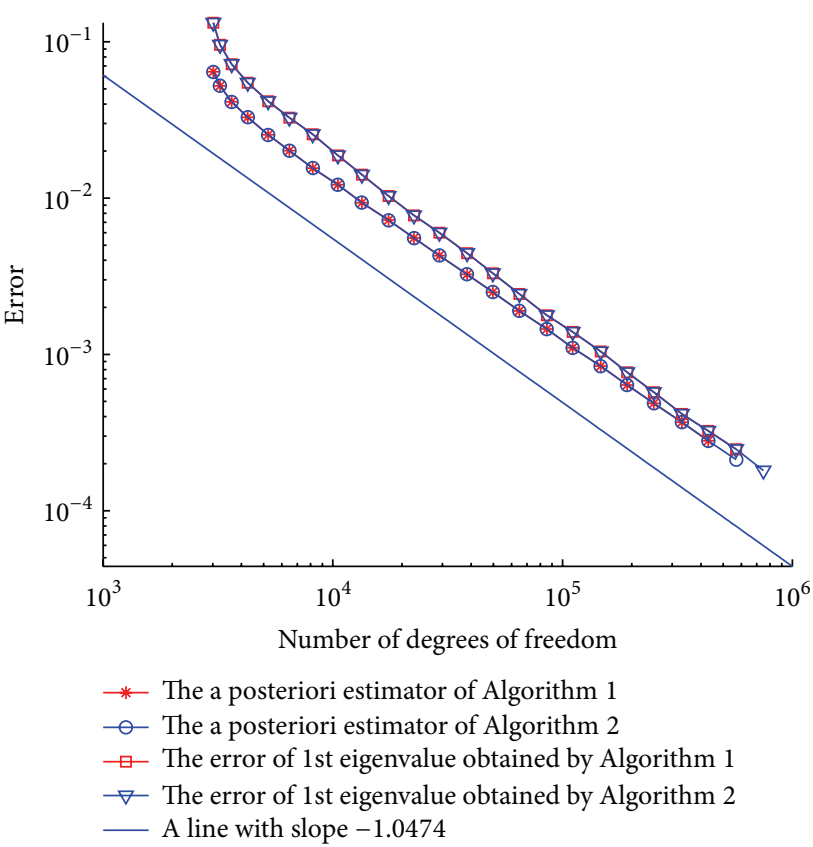

(a)

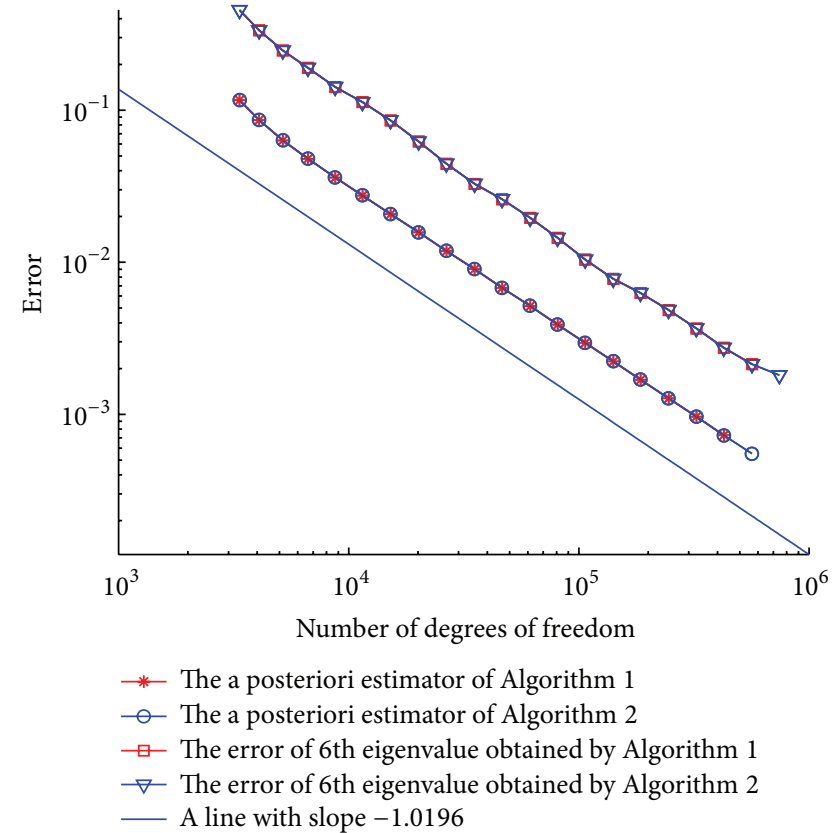

(b)

FIGURE 7: The error curves of two algorithms on slit domain.

But from Table 1, using Algorithm 11, we will spend much less time in the case of the same number of degrees of freedom but get the same accuracy to Algorithm 10. In addition, Algorithm 10, due to not having enough memory, can not proceed, while Algorithm 11 can have one more iteration; thus, more accurate numerical results will be obtained.

Example 2. We use Algorithms 10 and 11 to compute the approximate eigenvalues of $(1)$ on $\Omega=((0,2) \times(0,2)) \backslash([1,2] \times$ $\{1\}$ ) with a slit (see Figure 1(b)).
The first and sixth eigenvalues of (1) are $\lambda_{1} \approx$ 8.3713297112 and $\lambda_{6} \approx 30.536$ on this domain, respectively. The associated numerical results are presented in Table 2 and Figures 5, 6, and 7. Figure 7 show that the error curves of the first and sixth approximate eigenvalues and the curves of the associated a posteriori error estimators obtained by Algorithms 10 and 11 are approximately parallel to the line with slope -1 , respectively, which suffices to support our theory.

From Table 2, using Algorithm 11, compared with Algorithm 10, we can get the same accurate results in the case 
of the almost same degrees of freedom, but the CPU time is significantly decreased.

Remark 13. Based on the work of [30], we would like to believe that $\lambda^{h_{l}}$ and Rayleigh quotient $a\left(I_{h_{l}}^{c} u^{h_{l}}, I_{h_{l}}^{c} u^{h_{l}}\right) / b$ $\left(I_{h_{l}}^{c} u^{h_{l}}, I_{h_{l}}^{c} u^{h_{l}}\right)$ of $I_{h_{l}}^{c} u^{h_{l}}$ are the lower and upper bounds of the exact eigenvalue $\lambda$, respectively. To see this point, the numerical results of Tables 1 and 2 also illustrate that the $\mathrm{C}-\mathrm{R}$ element eigenvalues approximate the exact ones of the Laplace operator from below. Thus, we can establish iterative control condition by computing $\lambda^{h_{l}}$ and $a\left(I_{h_{l}}^{c} u^{h_{l}}, I_{h_{l}}^{c} u^{h_{l}}\right) /$ $b\left(I_{h_{l}}^{c} u^{h_{l}}, I_{h_{l}}^{c} u^{h_{l}}\right)$ for the two algorithms.

Remark 14. For Algorithm 12, by calculating, in the case of the almost same number of degrees of freedom, we can get the same accurate results to Algorithm 11, and CPU time is almost the same; thus, we do not list the associated numerical results in this paper.

\section{Conflict of Interests}

The authors declare that there is no conflict of interests regarding the publication of this paper.

\section{Acknowledgments}

This work was supported by National Natural Science Foundation of China (Grant no. 11161012) and Science and Technology Foundation of Guizhou Province of China (no. [2011]2111). The authors cordially thank the referees and the editor for their valuable comments and suggestions that led to the improvement of this paper.

\section{References}

[1] W. Dahmen, T. Rohwedder, R. Schneider, and A. Zeiser, "Adaptive eigenvalue computation: complexity estimates," Numerische Mathematik, vol. 110, no. 3, pp. 277-312, 2008.

[2] X. Dai, J. Xu, and A. Zhou, "Convergence and optimal complexity of adaptive finite element eigenvalue computations," Numerische Mathematik, vol. 110, no. 3, pp. 313-355, 2008.

[3] V. Heuveline and R. Rannacher, "A posteriori error control for finite approximations of elliptic eigenvalue problems," Advances in Computational Mathematics, vol. 15, no. 1-4, pp. 107-138, 2001.

[4] J. Han and Y. Yang, "A class of spectral element methods and its a priori/a posteriori error estimates for 2nd-order elliptic eigenvalue problems," Abstract and Applied Analysis, vol. 2013, Article ID 262010, 14 pages, 2013.

[5] D. Mao, L. Shen, and A. Zhou, "Adaptive finite element algorithms for eigenvalue problems based on local averaging type a posteriori error estimates," Advances in Computational Mathematics, vol. 25, no. 1-3, pp. 135-160, 2006.

[6] V. Mehrmann and A. Miedlar, "Adaptive computation of smallest eigenvalues of self-adjoint elliptic partial differential equations," Numerical Linear Algebra with Applications, vol. 18, no. 3, pp. 387-409, 2011.

[7] T. Rohwedder, R. Schneider, and A. Zeiser, "Perturbed preconditioned inverse iteration for operator eigenvalue problems with applications to adaptive wavelet discretization," Advances in Computational Mathematics, vol. 34, no. 1, pp. 43-66, 2011.

[8] Y. Yang, Y. Zhang, and H. Bi, "Multigrid discretization and iterative algorithm for mixed variational formulation of the eigenvalue problem of electric field," Abstract and Applied Analysis, vol. 2012, Article ID 190768, 25 pages, 2012.

[9] H. Bi, Y. Yang, and H. Li, "Local and parallel finite element discretizations for eigenvalue problems," SIAM Journal on Scientific Computing, vol. 35, no. 6, pp. 2575-2597, 2013.

[10] H. Li and Y. Yang, "The adaptive finite element method based on multi-scale discretizations for eigenvalue problems," Computers \& Mathematics with Applications, vol. 65, no. 7, pp. 1086-1102, 2013.

[11] Y. Yang and H. Bi, "Two-grid finite element discretization schemes based on shifted-inverse power method for elliptic eigenvalue problems," SIAM Journal on Numerical Analysis, vol. 49, no. 4, pp. 1602-1624, 2011.

[12] M. Crouzeix and P.-A. Raviart, "Conforming and nonconforming finite element methods for solving the stationary Stokes equations. I," RAIRO-Analyse Numérique, vol. 7, no. 3, pp. 3375, 1973.

[13] R. G. Durán, L. Gastaldi, and C. Padra, "A posteriori error estimators for mixed approximations of eigenvalue problems," Mathematical Models \& Methods in Applied Sciences, vol. 9, no. 8, pp. 1165-1178, 1999.

[14] Y. Li, "A posteriori error analysis of nonconforming methods for the eigenvalue problem," Journal of Systems Science \& Complexity, vol. 22, no. 3, pp. 495-502, 2009.

[15] C. Lovadina, M. Lyly, and R. Stenberg, "A posteriori estimates for the Stokes eigenvalue problem," Numerical Methods for Partial Differential Equations, vol. 25, no. 1, pp. 244-257, 2009.

[16] A. D. Russo and A. E. Alonso, "A posteriori error estimates for nonconforming approximations of Steklov eigenvalue problems," Computers \& Mathematics with Applications, vol. 62, no. 11, pp. 4100-4117, 2011.

[17] M. G. Armentano and R. G. Durán, "Asymptotic lower bounds for eigenvalues by nonconforming finite element methods," Electronic Transactions on Numerical Analysis, vol. 17, pp. 93101, 2004

[18] L. Chen, iFEM: An Innovation Finite Element Methods Package in MATLAB, 2008.

[19] I. Babuška and J. Osborn, "Eigenvalue problems," in Handbook of Numerical Analysis: Finite Element Methods (Part 1), P. G. Ciarlet and J. L. Lions, Eds., vol. 2, pp. 641-787, North-Holland, Amsterdam, The Netherlands, 1991.

[20] S. C. Brenner and L. R. Scott, The Mathematical Theory of Finite Element Methods, vol. 15 of Texts in Applied Mathematics, Springer, New York, NY, USA, 2nd edition, 2002.

[21] F. Chatelin, Spectral Approximation of Linear Operators, Computer Science and Applied Mathematics, Academic Press, New York, NY, USA, 1983.

[22] P. G. Ciarlet, "Basic error estimates for elliptic problems," in Handbook of Numerical Analysis: Finite Element Methods (Part 1), P. G. Ciarlet and J. L. Lions, Eds., vol. 2, pp. 17-351, NorthHolland, Amsterdam, The Netherlands, 1991.

[23] Y. Yang, Z. Zhang, and F. Lin, "Eigenvalue approximation from below using non-conforming finite elements," Science China. Mathematics, vol. 53, no. 1, pp. 137-150, 2010.

[24] Y. Yang, H. Bi, and Y. Yu, "The convergence and error estimates of the shifted inverse iteration based on multigrid discretizations for eigenvalue problems," submitted to SIAM Journal on Numerical Analysis. 
[25] A. Agouzal, "A posteriori error estimator for finite element discretizations of quasi-Newtonian Stokes flows," International Journal of Numerical Analysis and Modeling, vol. 2, no. 2, pp. 221-239, 2005.

[26] C. Carstensen, J. Hu, and A. Orlando, "Framework for the a posteriori error analysis of nonconforming finite elements," SIAM Journal on Numerical Analysis, vol. 45, no. 1, pp. 68-82, 2007.

[27] C. Carstensen and J. Hu, "A unifying theory of a posteriori error control for nonconforming finite element methods," Numerische Mathematik, vol. 107, no. 3, pp. 473-502, 2007.

[28] L. Wang and X. Xu, Foundation of Mathematics in Finite Element Methods, Science Press, Beijing, China, 2004 (Chinese).

[29] L. N. Trefethen and D. Bau III, Numerical Linear Algebra, Society for Industrial and Applied Mathematics, Philadelphia, Pa, USA, 1997.

[30] Y. Yang, J. Han, H. Bi, and Y. Yu, “The lower/upper bound property of the nonoconforming Crouzeix-Raviart element eigenvalues on adaptive meshes," Journal of Scientific Computing, 2014. 


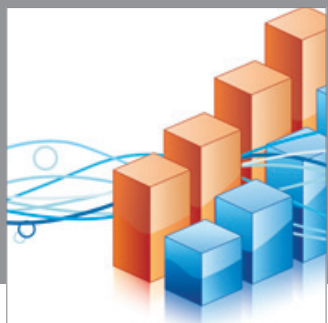

Advances in

Operations Research

mansans

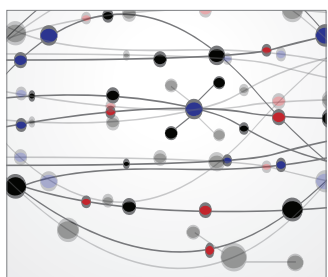

The Scientific World Journal
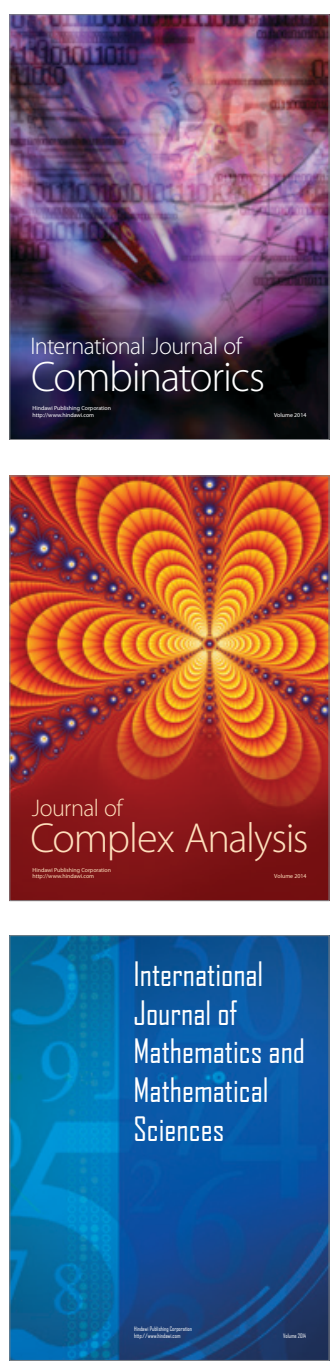
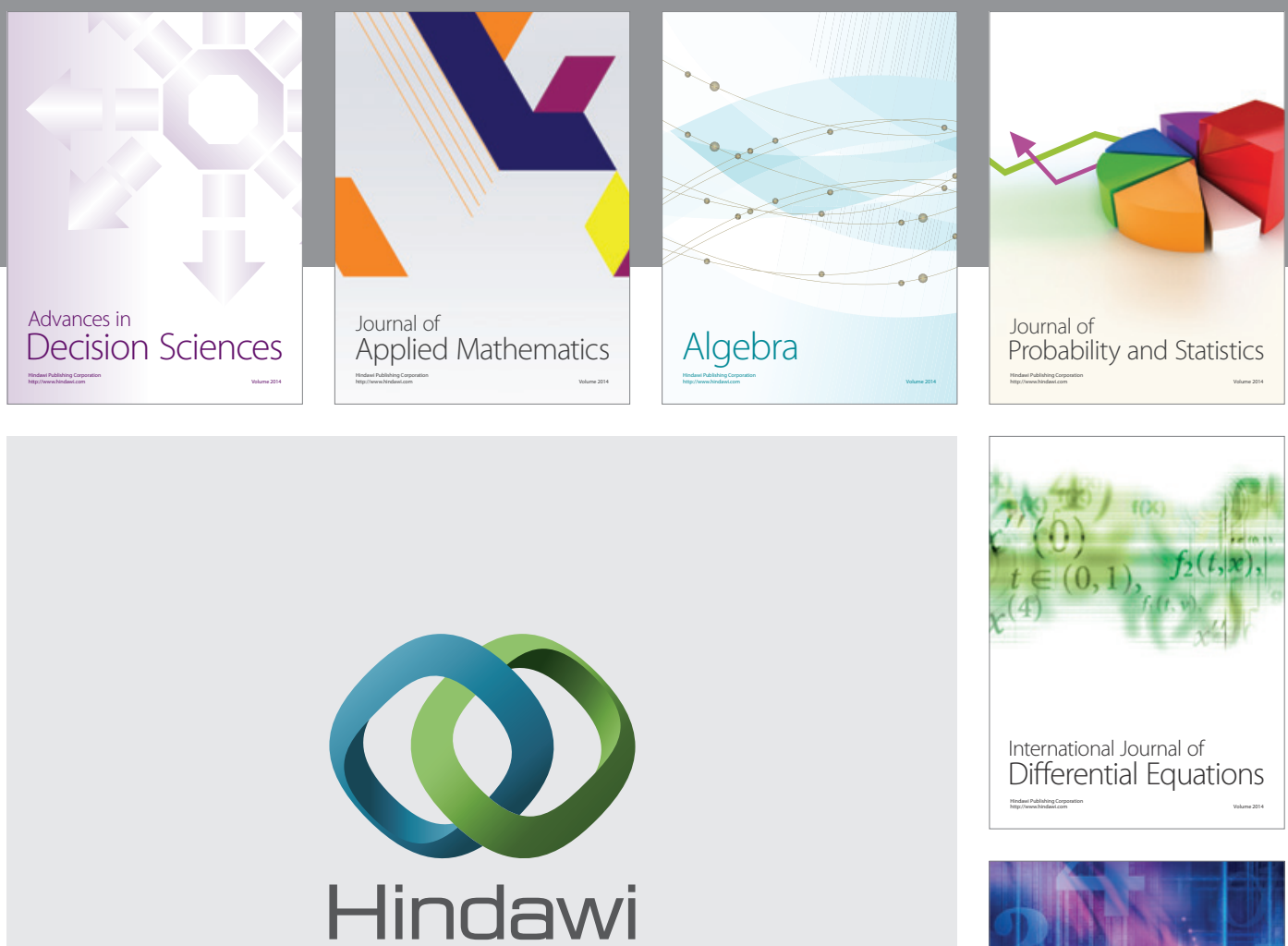

Submit your manuscripts at http://www.hindawi.com
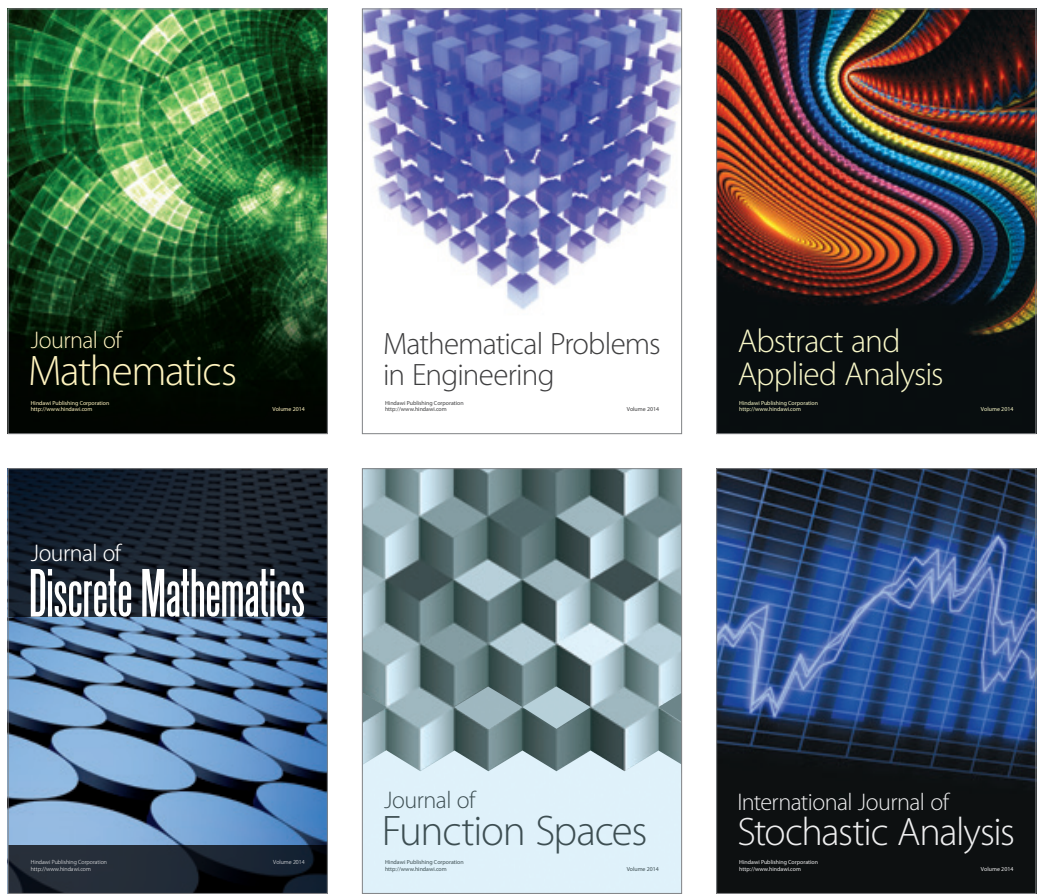

Journal of

Function Spaces

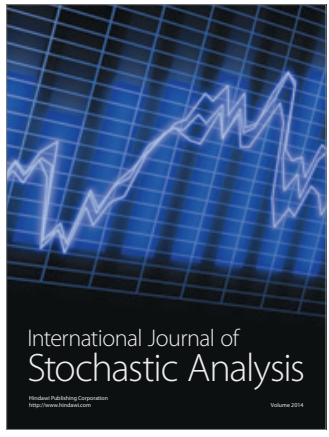

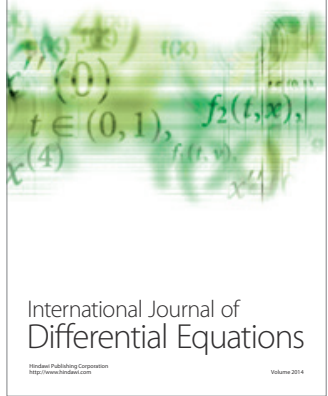
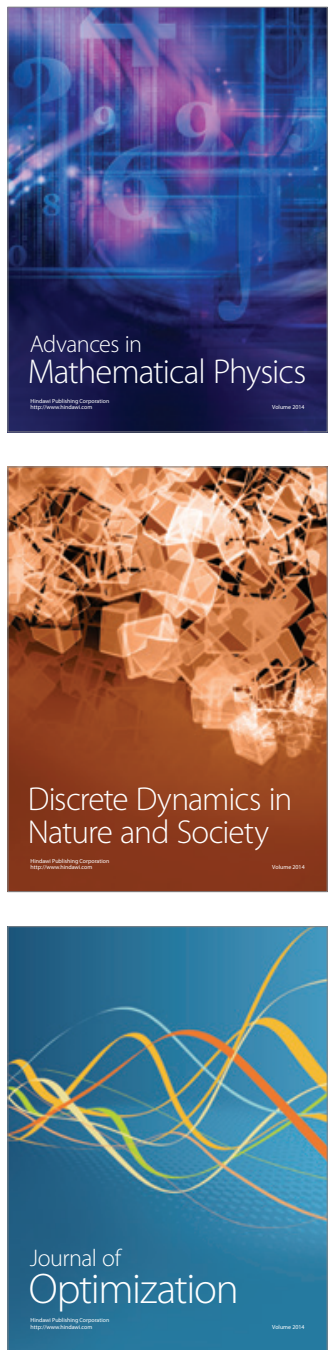